\title{
On Secrecy Rate Analysis of MIMO Wiretap Channels Driven by Finite-Alphabet Input
}

\author{
Shafi Bashar, Student Member, IEEE, Zhi Ding, Fellow, IEEE, and Chengshan \\ Xiao Fellow, IEEE
}

\begin{abstract}
This work investigates the effect of finite-alphabet input constraint on the secrecy rate of a multiantenna wiretap channel. Existing works have characterized maximum achievable secrecy rate or secrecy capacity for single and multiple antenna systems based on Gaussian source signals and secrecy code. Despite the impracticality of Gaussian sources, the compact closed-form expression of mutual information between linear channel Gaussian input and corresponding output has led to broad use of the Gaussian input assumption in physical secrecy analysis. For practical considerations, we study the effect of finite discrete-constellation on the achievable secrecy rate of multiple-antenna wire-tap channels. Our proposed precoding scheme converts the underlying multi-antenna system into a bank of parallel channels. Based on this precoding strategy, we develop a decentralized power allocation algorithm based on dual decomposition to maximize the achievable secrecy rate. In addition, we analyze the achievable secrecy rate for finite-alphabet inputs in low and high SNR regions. Our results demonstrate substantial difference in secrecy rate between systems given finite-alphabet inputs and systems with Gaussian inputs.
\end{abstract}

\section{Index Terms}

Wiretap channel, eavesdropping, information-theoretic security, secrecy rate, finite-alphabet input.

S. Bashar and Z. Ding are with the Department of Electrical and Computer Engineering, Univ. of California, Davis, CA, 95616, USA. e-mail: \{shafiab, ding\}@ece.ucdavis.edu.

C. Xiao is with the Department of Electrical and Computer Engineering, Missouri University of Science and Technology, Rolla, MO, 65409, USA. e-mail: xiaoc@mst.edu.

This material is based upon work supported by the National Science Foundation under Grants No. 0520126. 


\section{INTRODUCTION}

Wireless communications, with increasing coverage and applications, are vulnerable to potential security compromises such as passive eavesdropping and active jamming. Traditionally, network planners have relegated system security considerations to higher network layers of the OSI protocol stack through authentication and cryptography. However, in recent years, there have been growing research interests in the security analysis of wireless systems from a physical layer and information theoretic perspective. In a wiretap channel environment originally introduced by Wyner [?], a sender "Alice" wishes to transmit a secret message to the intended receiver "Bob" in the presence of a passive eavesdropper "Eve". Wyner [?] showed that when the Aliceto-Eve channel is degraded from the Alice-to-Bob channel, Alice can encode and send secure messages to the destination at a non-zero secrecy rate. In [?], a generalization for the nondegraded broadcast channel is proposed, and in [?], secrecy capacity of a Gaussian wiretap channel is shown to be achievable by adopting a random Gaussian codebook. In [?], the secrecy capacity of a multi-antenna Gaussian wiretap channel is shown to be achievable using a suitable input covariance matrix and by encoding the message using a Gaussian random codebook.

For both single- and multi-antenna Gaussian wiretap channels, the codebook that achieves secrecy capacity turns out to be Gaussian. However, such codebooks are not implementable in practice. In real world systems, input codebook consists of finite set of equi-probable constellation points (e.g. $M$-QAM, $M$-PAM etc.). Therefore, in contrast to the Gaussian codebook, practical wiretap codes must consist of finite-alphabet symbols. Because of this constraint, the achievable secrecy rate for a finite-alphabet input scenario would differ from the secrecy rate achieved by a Gaussian codebook.

A recent work [?] considered the effect of $M$-PAM input on the secrecy rate of a Gaussian wiretap channel and provided the necessary condition for power allocation to maximize the achievable secrecy rate. In [?], results were also extended to the case of parallel Gaussian wiretap channels. In [?], we have investigated the effect of finite-alphabet input on the ergodic secrecy rate of a multiple-input single-output and single-eavesdropper (MISOSE) system. To continue our progresses in this work, we investigate the effect of finite-alphabet input in a more general setting of a multiple-input multiple-output and multiple-eavesdropper (MIMOME) system. The specific contributions of the work are summarized below : 
- In order to quantify the effect of finite-alphabet input on MIMOME systems, we propose the application of precoding matrix to transform the MIMOME channel into a bank of parallel channels.

- We propose a power allocation optimization framework based on decentralized dual decomposition technique to maximize the achievable secrecy rate of MIMOME systems with an arbitrary but known input distribution.

- We provide secrecy rate analysis of MIMOME systems with finite-alphabet inputs at low and high SNR regions. Our findings suggest that similar to the Gaussian wiretap channel, proper transmission power should be diverted at high signal-to-noise ratio (SNR) in case of finite-alphabet input albeit with different effect.

We organize the rest of the paper as follows. We begin with the system model in section II In section III we propose a linear precoding scheme that transforms the MIMOME wiretap channel into a set of parallel channels. Based on this precoding scheme, we reformulate the secrecy rate problem for an arbitrary input distribution. In section IV, we develop a decentralized power allocation algorithm based on dual decomposition that maximizes the achievable secrecy rate for an arbitrary distribution. In section $\mathrm{V}$, we further consider the special case of Gaussian input and present a modified water-filling power allocation strategy by considering the secrecy constraint. We then extend the modified water-filling power allocation scheme to analyze the secrecy rate for an arbitrary input distribution in both low and high SNR regions. In section VI we present numerical results before concluding with section VII.

\section{PRELIMINARIES AND SySTEM DESCRIPTION}

Throughout this work, we use notations $\operatorname{tr}($.$) , \operatorname{det}($.$) and superscript \{\cdot\}^{H}$, respectively, to denote the trace, the determinant, and the conjugate transpose of a matrix.

\section{A. System Model}

We consider a MIMO (multiple-input multiple-output) wiretap system model in which the transmitter (Alice), the intended receiver (Bob), and the passive eavesdropper (Eve), respectively, have $m_{a}$ (transmit), $m_{b}$ (receive), and $m_{e}$ (eavesdrop) antennas. Denote the received signals at 
Bob and Eve as $\mathbf{y}_{b}$ and $\mathbf{y}_{e}$, respectively. Their received signals are written as

$$
\begin{aligned}
& \mathbf{y}_{b}=\mathbf{H}_{b} \mathbf{x}+\mathbf{n}_{b} \\
& \mathbf{y}_{e}=\mathbf{H}_{e} \mathbf{x}+\mathbf{n}_{e}
\end{aligned}
$$

where $\mathbf{H}_{b} \in \mathbb{C}^{m_{b} \times m_{a}}$ and $\mathbf{H}_{e} \in \mathbb{C}^{m_{e} \times m_{a}}$ denote, respectively, the flat-fading MIMO channels, from Alice-to-Bob and from Alice-to-Eve. The noise $\mathbf{n}_{b} \in \mathbb{C}^{m_{b}}$ and $\mathbf{n}_{e} \in \mathbb{C}^{m_{e}}$ are zero-mean identity matrix variance complex Gaussian random vectors independent of each other. The data signal is $\mathbf{x} \in \mathbb{C}^{m_{a}}$ transmitted by Alice in the form of $\mathbf{x}=\mathbf{W s}$, in which $\mathbf{W}$ is a linear precoding matrix. We denote $\mathbf{s}$ as a random vector with zero mean entries and identity correlation matrix. We constrain the total transmission power by a peak level $P_{T}$, i.e., $\operatorname{tr}\left\{\mathbf{K}_{\mathbf{x}}\right\} \leq P_{T}$, where $\mathbf{K}_{\mathbf{x}}=\mathbb{E}\left\{\mathbf{x x}^{H}\right\}$ is the covariance matrix of the transmitted signal vector.

The secrecy capacity of the above system is achievable by using a Gaussian random codebook [?]. First, let $A \succeq B$ denote that $A-B$ is non-negative definite. The secrecy capacity is the solution of the following optimization problem

$$
\begin{aligned}
& \underset{\mathbf{K}_{\mathbf{x}}}{\operatorname{maximize}} \log \operatorname{det}\left(\mathbf{I}+\mathbf{H}_{b} \mathbf{K}_{\mathbf{x}} \mathbf{H}_{b}^{H}\right)-\log \operatorname{det}\left(\mathbf{I}+\mathbf{H}_{e} \mathbf{K}_{\mathbf{x}} \mathbf{H}_{e}^{H}\right) \\
& \text { subject to : } \quad \mathbf{K}_{\mathbf{x}} \succeq 0, \mathbf{K}_{\mathbf{x}}=\mathbf{K}_{\mathbf{x}}^{H} \\
& \qquad \operatorname{tr}\left(\mathbf{K}_{\mathbf{x}}\right) \leq P_{T} .
\end{aligned}
$$

In order to realize an achievable secrecy rate for an arbitrary input signaling, we will generalize the objective function. Instead of the optimal Gaussian signaling as used in the above optimization problem, we replace the objective function with the more general form of $I\left(\mathbf{x} ; \mathbf{y}_{b}\right)-I\left(\mathbf{x} ; \mathbf{y}_{e}\right)$, where $I(\mathbf{x} ; \mathbf{y})$ represents the mutual information between the input and output vectors $\mathbf{x}$ and $\mathbf{y}$.

The optimization problem described in (3) is a non-convex optimization problem (except for the special case of $m_{b}=m_{e}=1$ [?], [?]). Even for simple cases, the objective function possesses a number of local maxima. Therefore, the optimum value of $\mathbf{K}_{x}$ is in general not known. In particular, for an arbitrary input signaling without any closed-form mutual information, solving the above optimization problem can be a difficult task. Thus, instead of solving the above optimization problem, we consider a particular linear precoding scheme similar to the one provided in [?]. The proposed linear precoding scheme first transforms the MIMOME channel into a bank of parallel channels. This step allows us to gain a better understanding of the effect of finite-alphabet input on the secrecy of the system. It enables us to gain better insights 
into future system implementation. Such linear precoding scheme is justifiable from practical system implementation perspective. In addition, at high SNR, such precoding scheme is known to achieve the capacity of a MIMOME system [?].

\section{B. Preliminaries}

Definition 1: Similar to [?], we define the following subspaces

$$
\begin{aligned}
\mathcal{S}_{b} & =\operatorname{null}\left(\mathbf{H}_{b}\right)^{\perp} \cap \operatorname{null}\left(\mathbf{H}_{e}\right) \\
\mathcal{S}_{b, e} & =\operatorname{null}\left(\mathbf{H}_{b}\right)^{\perp} \cap \operatorname{null}\left(\mathbf{H}_{e}\right)^{\perp} \\
\mathcal{S}_{e} & =\operatorname{null}\left(\mathbf{H}_{b}\right) \cap \operatorname{null}\left(\mathbf{H}_{e}\right)^{\perp} \\
\mathcal{S}_{n} & =\operatorname{null}\left(\mathbf{H}_{b}\right) \cap \operatorname{null}\left(\mathbf{H}_{e}\right) .
\end{aligned}
$$

In fact, subspace $\mathcal{S}_{b}$ corresponds to the class of input with non-zero gain towards the direction of Bob only. Subspace $\mathcal{S}_{b, e}$ corresponds to the class of input with non-zero gain in the direction of both Bob and Eve. $\mathcal{S}_{e}$ corresponds to the class of input with non-zero gain in the direction of Eve only. Finally, $\mathcal{S}_{n}$ is the subspace with non-zero gain in the direction occupied by neither Bob nor Eve. Define $k=\operatorname{rank}\left(\left[\begin{array}{cc}\mathbf{H}_{b}^{H} & \mathbf{H}_{e}^{H}\end{array}\right]^{H}\right)$ and hence $\operatorname{dim}\left(\mathcal{S}_{n}\right)=m_{a}-k$. In addition, we define, $r=\operatorname{dim}\left(\mathcal{S}_{b}\right)$ and $s=\operatorname{dim}\left(\mathcal{S}_{r, e}\right)$. Therefore, $\operatorname{dim}\left(\mathcal{S}_{e}\right)=k-r-s$.

Definition 2: We recall the following definition of generalized singular value decomposition (GSVD) [?], [?] that we will use for our analysis. The GSVD of the pair $\left(\mathbf{H}_{b}, \mathbf{H}_{e}\right)$ takes the following form

$$
\begin{gathered}
\mathbf{H}_{b}=\boldsymbol{\Psi}_{b} \boldsymbol{\Sigma}_{b}\left[\begin{array}{cc}
k & m_{a}-k \\
\boldsymbol{\Omega}^{-1} & \mathbf{0}
\end{array}\right] \boldsymbol{\Psi}_{a}^{H} \\
\mathbf{H}_{e}=\boldsymbol{\Psi}_{e} \boldsymbol{\Sigma}_{e} \quad\left[\begin{array}{cc}
\mathrm{m}_{a}-k \\
\boldsymbol{\Omega}^{-1} & \mathbf{0}
\end{array}\right] \boldsymbol{\Psi}_{a}^{H}
\end{gathered}
$$

where $\boldsymbol{\Psi}_{a} \in \mathbb{C}^{m_{a} \times m_{a}}, \boldsymbol{\Psi}_{b} \in \mathbb{C}^{m_{b} \times m_{b}}$, and $\boldsymbol{\Psi}_{e} \in \mathbb{C}^{m_{e} \times m_{e}}$ are unitary matrices. $\boldsymbol{\Omega} \in \mathbb{C}^{k \times k}$ is a non-singular matrix. $\Sigma_{b} \in \mathbb{C}^{m_{b} \times k}$ and $\Sigma_{e} \in \mathbb{C}^{m_{e} \times k}$ have the following form 


$$
\begin{aligned}
& \boldsymbol{\Sigma}_{b}=m_{s} m_{b}-r-s\left[\begin{array}{ccc}
k-r-s & s & r \\
\mathbf{0} & \mathbf{0} & \mathbf{0} \\
\mathbf{0} & \mathbf{D}_{b} & \mathbf{0} \\
\mathbf{0} & \mathbf{0} & \mathbf{I}
\end{array}\right] \\
& \boldsymbol{\Sigma}_{e} \operatorname{men}_{m_{e}-k+r}\left[\begin{array}{ccc}
k-r-s & s & r \\
\mathbf{I} & \mathbf{0} & \mathbf{0} \\
\mathbf{0} & \mathbf{D}_{e} & \mathbf{0} \\
\mathbf{0} & \mathbf{0} & \mathbf{0}
\end{array}\right]
\end{aligned}
$$

$\mathbf{D}_{b}=\operatorname{diag}\left(\left\{b_{1}, \ldots, b_{s}\right\}\right)$ and $\mathbf{D}_{e}=\operatorname{diag}\left(\left\{e_{1}, \ldots, e_{s}\right\}\right)$ are diagonal real matrices with dimension $s \times s$. The diagonal entries of $\mathbf{D}_{b}$ and $\mathbf{D}_{e}$ are arranged in the following orders:

$$
\begin{gathered}
0<b_{1} \leq \ldots b_{s}<1 \\
1>e_{1} \geq \ldots \geq e_{s}>0
\end{gathered}
$$

and

$$
b_{i}^{2}+e_{i}^{2}=1, \text { for } i=1, \ldots, s
$$

\section{Secrecy Rate Problem Formulation}

\section{A. Proposed Precoding Strategy}

Given the above two definitions in hand, we propose the following precoding matrix

$$
\mathbf{W}=\Psi_{a} \mathbf{B P}^{1 / 2}
$$

where, $\mathbf{B}$ is defined as follows

$$
\mathbf{B}={ }_{m_{a}-k}^{k}\left[\begin{array}{cc}
\boldsymbol{\Omega} & m_{a}-k \\
\mathbf{0} & \mathbf{0}
\end{array}\right]
$$

$P=\operatorname{diag}\left(\left\{p_{1}, \ldots, p_{m_{a}}\right\}\right)$ is a diagonal power allocation matrix. The proposed precoding matrix in Eq. (4) is similar to the precoding strategy defined in [?]. However, unlike the precoding 


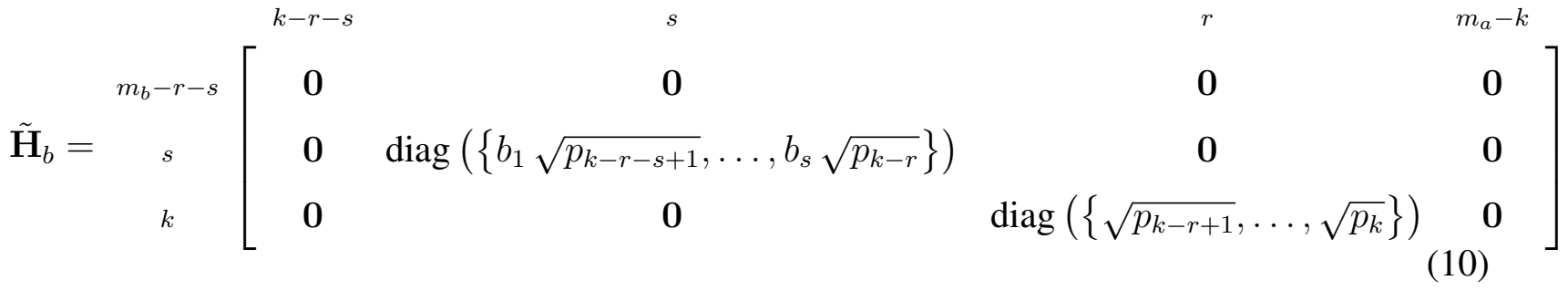

$$
\begin{aligned}
& \tilde{\mathbf{H}}_{e}={ }_{m_{e}-k+r}^{k-r-s}\left[\begin{array}{ccc}
k-r-s & s & m_{a}-k+r \\
\operatorname{diag}\left(\left\{\sqrt{p_{1}}, \ldots, \sqrt{p_{k-r-s}}\right\}\right) & \mathbf{0} & \mathbf{0} \\
\mathbf{0} & \operatorname{diag}\left(\left\{e_{1} \sqrt{p_{k-r-s+1}}, \ldots, e_{s} \sqrt{p_{k-r}}\right\}\right) & \mathbf{0} \\
\mathbf{0} & \mathbf{0} & \mathbf{0}
\end{array}\right]
\end{aligned}
$$

scheme in [?], the diagonal elemens $\left\{p_{i}\right\}$ in the power allocaion matrix can have different values. As will be evident in the subsequent sections, such differentiation is extremely important due to the finite nautre of the input constellations considered in this work.

By using the above precoding strategy, the system equations of (1), (2) become

$$
\begin{aligned}
& \mathbf{y}_{b}=\boldsymbol{\Psi}_{b} \boldsymbol{\Sigma}_{b}\left[\begin{array}{cc}
k & m_{a}-k \\
\mathbf{I} & \mathbf{0}
\end{array}\right] \mathbf{P}^{1 / 2} \mathbf{s}+\mathbf{n}_{b} \\
& \mathbf{y}_{e}=\boldsymbol{\Psi}_{e} \boldsymbol{\Sigma}_{e}\left[\begin{array}{cc}
m_{a}-k \\
\mathbf{I} & \mathbf{0}
\end{array}\right] \mathbf{P}^{1 / 2} \mathbf{s}+\mathbf{n}_{e} .
\end{aligned}
$$

Pre-multiplying (6) and (7) with $\Psi_{b}^{H}$ and $\Psi_{e}^{H}$, respectively, we have the following equivalent equations

$$
\begin{aligned}
\tilde{\mathbf{y}}_{b} & =\tilde{\mathbf{H}}_{b} \mathbf{s}+\tilde{\mathbf{n}}_{b} \\
\tilde{\mathbf{y}}_{e} & =\tilde{\mathbf{H}}_{e} \mathbf{s}+\tilde{\mathbf{n}}_{e}
\end{aligned}
$$

where we use the notations $\tilde{\mathbf{y}}_{b}=\Psi_{b}^{H} \mathbf{y}_{b}, \tilde{\mathbf{y}}_{e}=\Psi_{e}^{H} \mathbf{y}_{e}, \tilde{\mathbf{n}}_{b}=\Psi_{b}^{H} \mathbf{n}_{b}$ and $\tilde{\mathbf{n}}_{e}=\Psi_{e}^{H} \mathbf{n}_{e}$. The new equivalent channel matrices $\tilde{\mathbf{H}}_{b}=\boldsymbol{\Sigma}_{b}\left[\begin{array}{ll}\mathbf{I} & \mathbf{0}\end{array}\right] \mathbf{P}^{1 / 2}$ and $\tilde{\mathbf{H}}_{e}=\boldsymbol{\Sigma}_{e}\left[\begin{array}{ll}\mathbf{I} & \mathbf{0}\end{array}\right] \mathbf{P}^{1 / 2}$ are specified in Eqs. (10) and (11).

From Eqs. (10), (11), we observe that the new system equations (8), (9) in fact transform the MIMOME system (1), (2) into a bank of parallel channels. Fig. 11 shows the resulting 
parallel channel model. In this parallel channel model, input symbols $s_{1}, \ldots, s_{k-r-s}$ are only observed by Eve, symbols $s_{k-r-s+1}, \ldots, s_{k-r}$ are received by both Bob and Eve, whereas symbols $s_{k-r+1}, \ldots, s_{k}$ are only received by Bob. Finally, symbols $s_{k+1}, \ldots, s_{m_{a}}$ are lost by receivers of both Bob and Eve.

\section{B. Reformulation of Secrecy Rate Problem}

In this section, we relax the secrecy capacity problem of Eq. (3) using the precoding matrix presented in Sec. III-A. We generalize the problem into an achievable secrecy rate problem for arbitrary input distribution. To this end, we present the following proposition.

Proposition 1: Define $\mathcal{I}(\gamma)=I(s ; \sqrt{\gamma} s+n), \nu=k-r-s, \mathbf{p}=\left\{p_{1}, \ldots, p_{m_{a}}\right\},\left\{\omega_{i}\right\}=$ $\operatorname{diag}\left(\Omega^{H} \Omega\right)$. When input $\mathbf{s}$ of a MIMOME system is a random vector with zero mean entries and identity correlation matrix, by using the precoding matrix $\mathbf{W}$ defined in Eq. (4), we can achieve the following secrecy rate for an arbitrary distribution of $\mathbf{s}$

$$
\begin{aligned}
& \underset{\mathbf{p}}{\operatorname{maximize}} \sum_{i: b_{i}>e_{i}}\left[\mathcal{I}\left(\frac{b_{i}^{2}}{\omega_{\nu+i}} p_{\nu+i}\right)-\mathcal{I}\left(\frac{e_{i}^{2}}{\omega_{\nu+i}} p_{\nu+i}\right)\right]+\sum_{j=k-r+1}^{k} \mathcal{I}\left(\frac{1}{\omega_{j}} p_{j}\right) \\
& \text { subject to }: \quad \sum_{i: b_{i}>e_{i}} p_{\nu+i}+\sum_{j=k-r+1}^{k} p_{j} \leq P_{T}
\end{aligned}
$$

Proof: See Appendix A

We observe that, the application of the proposed precoding matrix can transform the MIMOME problem into a distributed secrecy rate problem for a bank of parallel channels. We note that, for a given alphabet set, in general the above optimization problem should be jointly optimized over the input probability distribution and the power allocation. However, practical modulation constellations are generally constrained to be equi-probable. Therefore, here we will consider equi-probable input alphabet and focus on power allocation optimization. Next we propose a power allocation algorithm to solve the above optimization problem.

\section{POWER ALLOCATION ALGORITHM FOR ARBITRARY INPUT DISTRIBUTION}

Even though the above optimization problem is convex for Gaussian input, for an arbitrary input distribution, this is in general not the case. In addition, the lack of closed-form expression for mutual information makes the problem even more difficult to solve. In order to find an efficient 
sub-optimal solution, we will revert to the decomposition technique. We note that, without the sum power constraint, the optimization problem (12) can be decoupled into a number of parallel problems each involving only one variable $p_{i}$. However, the sum power constraint compels us to solve a larger optimization problem jointly involving multiple variables. For problem such as (12) involving a complex constraint, a dual decomposition method [?], [?] based on the Lagrangian of the objective function enables us to readily decompose the problem into a number of parallel sub-problems each involving a single variable. These subproblems are linked through a master problem that updates the dual variable during each iterations of the subproblems. By introducing a dual variable $\mu$, we can write the Lagrangian of the optimization problem (12) by relaxing the coupling constraint as follows

$$
\begin{aligned}
& \underset{\mathbf{p}}{\operatorname{maximize}} \sum_{i: b_{i}>e_{i}}\left[\mathcal{I}\left(\frac{b_{i}^{2}}{\omega_{\nu+i}} p_{\nu+i}\right)-\mathcal{I}\left(\frac{e_{i}^{2}}{\omega_{\nu+i}} p_{\nu+i}\right)\right]+\sum_{j=k-r+1}^{k} \mathcal{I}\left(\frac{1}{\omega_{j}} p_{j}\right) \\
& \quad-\mu\left(\sum_{i: b_{i}>e_{i}} p_{\nu+i}+\sum_{j=k-r+1}^{k} p_{j}\right)+\mu P_{T}
\end{aligned}
$$

subject to: $p_{i} \geq 0$.

Note that, the above optimization problem is decoupled in terms of $p_{i}$. We obtain the following two subproblems.

Subproblem 1 : for all $i$ such that $b_{i}>e_{i}$

$$
\underset{p_{i} \geq 0}{\operatorname{maximize}}\left[\mathcal{I}\left(\frac{b_{i}^{2}}{\omega_{\nu+i}} p_{\nu+i}\right)-\mathcal{I}\left(\frac{e_{i}^{2}}{\omega_{\nu+i}} p_{\nu+i}\right)\right]-\mu p_{\nu+i}
$$

Subproblem 2 : for all $j=k-r+1, \ldots, k$

$$
\underset{p_{i} \geq 0}{\operatorname{maximize}} \mathcal{I}\left(\frac{1}{\omega_{j}} p_{j}\right)-\mu p_{j}
$$

Subproblems 1 and 2 are linked through the master problem which is the dual optimization problem of $(12)$. The master problem updates the value of the dual variables $\mu$. Let $p_{i}^{*}$ denote the solution found from the subproblems. The master problem can be written as follows

\section{Master Problem :}

$$
\begin{aligned}
& \underset{\mu \geq 0}{\operatorname{minimize}} \sum_{i: b_{i}>e_{i}}\left[\mathcal{I}\left(\frac{b_{i}^{2}}{\omega_{\nu+i}} p_{\nu+i}^{*}\right)-\mathcal{I}\left(\frac{e_{i}^{2}}{\omega_{\nu+i}} p_{\nu+i}^{*}\right)\right]+\sum_{j=k-r+1}^{k} \mathcal{I}\left(\frac{1}{\omega_{j}} p_{j}^{*}\right) \\
& \quad-\mu\left(\sum_{i: b_{i}>e_{i}} p_{\nu+i}^{*}+\sum_{j=k-r+1}^{k} p_{j}^{*}\right)+\mu P_{T} .
\end{aligned}
$$


Subproblem 2 is a convex problem and can be solve optimally. However, in general subproblem 1 is not convex. Even though subproblems 1 and 2 only involve single variable, in most situations, a closed form or analytic expression for the mutual information is not known for an arbitrary input distribution. In order to solve subproblems 1 and 2, we resort to a recent result on finitealphabet research [?], [?] that relates the mutual information and the minimum mean square error (MMSE) at the receiver through

$$
\frac{d \mathcal{I}(\rho)}{d \rho}=\operatorname{mmse}(\rho) .
$$

Note that the function "mmse" for different discrete constellations (e.g., $M$-PSK, $M$-QAM etc., where $M$ is the number of constellation points) has been given in [?]. Using Eq. (17), the optimum value of $p_{i}$ in subproblems 2 can be solved from the following equations

$$
\frac{1}{\omega_{j}} \text { mmse }\left(\frac{1}{\omega_{j}} p_{j}^{*}\right)-\mu=0, \text { for } j=k-r+1, \ldots, k \text {. }
$$

Eq. (18) can be further expressed as follows

$$
p_{j}^{*}=\omega_{j} \operatorname{mmse}^{-1}\left(\min \left\{1, \mu \omega_{j}\right\}\right), \text { for } j=k-r+1, \ldots, k
$$

where we used the fact that $\mathrm{mmse}^{-1}(1)=0$.

For subproblem 1, we can derive the following necessary condition for optimality

$$
\begin{gathered}
\frac{b_{i}^{2}}{\omega_{\nu+i}} \operatorname{mmse}\left(\frac{b_{i}^{2}}{\omega_{\nu+i}} p_{\nu+i}^{*}\right)-\frac{e_{i}^{2}}{\omega_{\nu+i}} \operatorname{mmse}\left(\frac{e_{i}^{2}}{\omega_{\nu+i}} p_{\nu+i}^{*}\right)-\mu=0 \\
\text { for } i: b_{i}>e_{i}
\end{gathered}
$$

Next, we propose a sufficient condition for the optimality of the solution of Eq. (20). In this regard, we define the following MMSE difference function

$$
f_{\mathrm{mmseD}}\left(p, b_{i}, e_{i}, \omega_{\nu+i}\right)=\frac{b_{i}^{2}}{\omega_{\nu+i}} \operatorname{mmse}\left(\frac{b_{i}^{2}}{\omega_{\nu+i}} p_{\nu+i}^{*}\right)-\frac{e_{i}^{2}}{\omega_{\nu+i}} \operatorname{mmse}\left(\frac{e_{i}^{2}}{\omega_{\nu+i}} p_{\nu+i}^{*}\right) .
$$

We note that, a similar condition has been proposed in [?] [see Theorem 6 in [?] for a parallel Gaussian wiretap channel with $M$-PAM inputs.

Proposition 2: If the MMSE difference function in Eq. (21) admits a unique zero $p^{\prime}$ and is strictly monotonically decreasing for $0 \leq p \leq p^{\prime}$, then the optimal solution $\left\{p_{\nu+i}^{*}\right\}$ of Eq. (20) can be given as follows

when $b_{i}^{2} \leq e_{i}^{2}$ 


$$
p_{\nu+i}^{*}=0
$$

when $b_{i}^{2}>e_{i}^{2}$

$$
\begin{aligned}
& p_{\nu+i}^{*}=p^{\prime} \quad \text { if } \mu=0 \\
& \frac{b_{i}^{2}}{\omega_{\nu+i}} \operatorname{mmse}\left(\frac{b_{i}^{2}}{\omega_{\nu+i}} p_{\nu+i}^{*}\right)-\frac{e_{i}^{2}}{\omega_{\nu+i}} \operatorname{mmse}\left(\frac{e_{i}^{2}}{\omega_{\nu+i}} p_{\nu+i}^{*}\right)=\mu, \quad 0<p<p^{\prime} \quad \text { if } \mu>0 .
\end{aligned}
$$

Proof: See Appendix B.

In following proposition, we describe a condition for the MMSE difference function to admit a unique zero solution.

Proposition 3: If $g(\rho)=\rho \operatorname{mmse}(\rho)$ is a strictly uni-modal function and $b_{i}>e_{i}$, then the MMSE difference function $f_{\mathrm{mmseD}}\left(p, b_{i}, e_{i}, \omega_{\nu+i}\right)$ admits a unique zero solution for $p>0$.

Proof: See Appendix C

In Figure 6, we present the plot of $g(\rho)=\rho \operatorname{mmse}(\rho)$ vs. $\rho$ for BPSK, QPSK, 16-QAM, and 64-QAM input constellations. We observe that $g(\rho)$ of all four constellations shows strictly unimodality in the region of interest. Furthermore, in Figures 7, 8, 9, and 10, we illustrate graphically the MMSE difference function $f_{\mathrm{mmseD}}(p, a)$ vs. power $p$ for various values of $a=e_{i}^{2} / b_{i}^{2}$. Here, we also assume $\omega_{\nu+i}=1$ without any loss of generality. We observe that, the MMSE difference function exhibits strictly monotonically decreasing behavior in the range $0 \leq p \leq p^{\prime}$ for all four constellations. Based on Propositions 2 and 3 , and Figures 6,10 , we see that the optimality criterion described in proposition 2 holds for common constellations of BPSK, QPSK, 16-QAM, and 64-QAM. Therefore, the solution of subproblem 1 in Eq. (19) will be unique for at least these four constellations.

The combined algorithm to solve the optimization problem (12) is presented in Algorithm (1).

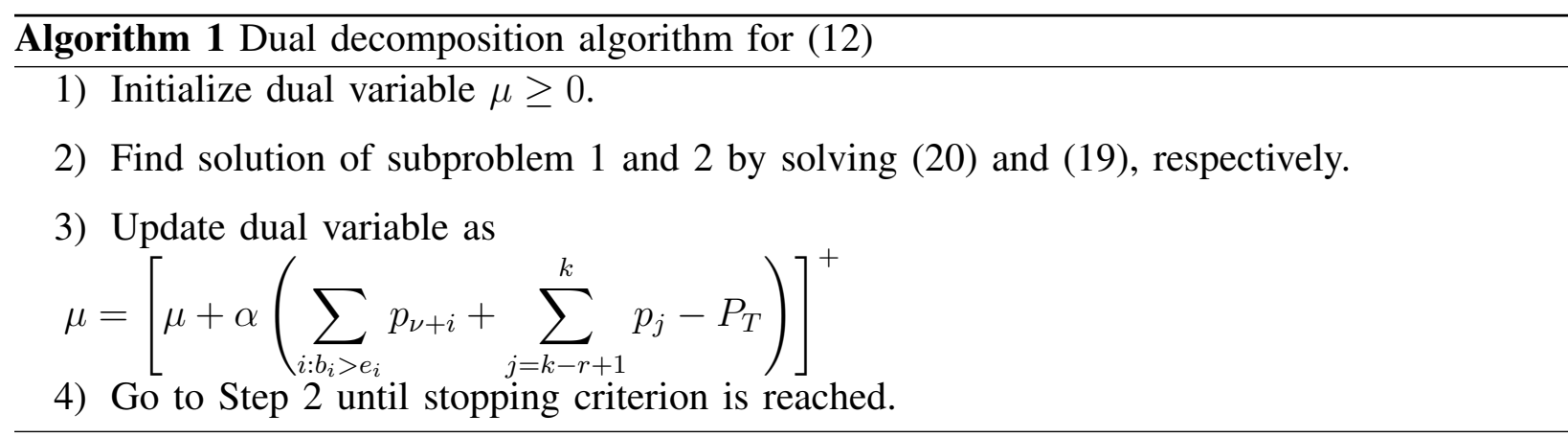

We note that the master problem in Eq. (16) is differentiable with respect to the dual variable $\mu$. Therefore, in Step 3 of the above algorithm, we use the gradient method to update the dual 
variable. Here, the parameter $\alpha>0$ denotes an appropriate stepsize, which can be either a constant or time-varying. In our simulation, we choose a fixed stepsize $\alpha$. If the stepsize is sufficiently small, then the solution of the above algorithm will converge to the solution of the optimal dual variable $\mu^{*}$. A detail description on the choice of stepsize and stopping criterion can be found in [?], [?] and the references therein. If the original optimization problem in Eq. (12) is convex, then the duality gap will be zero. Therefore, the solution of the dual problem in Eq. (16) will also provide the optimal solution of the original problem. However, if the original problem is not convex, then there exists a positive duality gap and the solution of Eq. (16) will be a suboptimal solution of the original problem.

\section{Secrecy Rate Analysis of Mimome System}

\section{A. Gaussian Input Case}

In this section, we will present the power allocation problem for the special case of Gaussian input distribution based on the framework considered above. Even though the result for Gaussian input is well known [?], [?], [?], results in this section will provide additional insight for the finite-alphabet input scenarios to be considered later.

For Gaussian input, the MMSE equation is simply

$$
\operatorname{mmse}(\gamma)=\frac{1}{1+\gamma}
$$

Let us denote $\left\{p_{i}^{\mathrm{g}}\right\}$ as the optimum power allocation for Gaussian input. Based on Eq. (22), solutions of subproblem 1 can be found by solving the following equation

$$
\frac{b_{i}^{2} e_{i}^{2}}{b_{i}^{2}-e_{i}^{2}} \frac{1}{\omega_{\nu+1}}\left(p_{\nu+1}^{\mathrm{g}}\right)^{2}+\frac{1}{b_{i}^{2}-e_{i}^{2}} p_{\nu+1}^{\mathrm{g}}+\left[\frac{\omega_{\nu+1}}{b_{i}^{2}-e_{i}^{2}}-\frac{1}{\mu}\right]=0 .
$$

Thus, the optimal power allocation is

$$
p_{\nu+i}^{\mathrm{g}}=\left\{\begin{array}{ll}
0, & \text { if } \frac{1}{\mu} \leq \frac{\omega_{\nu+i}}{b_{i}^{2}-e_{i}^{2}} \\
\frac{1}{2}\left[\sqrt{\left(\frac{\omega_{\nu+i}}{b_{i}^{2} e_{i}^{2}}\right)^{2}+4 \frac{\omega_{\nu+i}}{b_{i}^{2} e_{i}^{2}}\left(b_{i}^{2}-e_{i}^{2}\right)\left(\frac{1}{\mu}-\frac{\omega_{\nu+i}}{b_{i}^{2}-e_{i}^{2}}\right)}-\frac{\omega_{\nu+i}}{b_{i}^{2} e_{i}^{2}}\right], & \text { if } \frac{1}{\mu}>\frac{\omega_{\nu+i}}{b_{i}^{2}-e_{i}^{2}}
\end{array} .\right.
$$

Similarly, based on Eq. (22), solution of subproblem 2 can be found as

$$
p_{i}^{\mathrm{g}}=\left\{\begin{array}{ll}
0, & \text { if } \frac{1}{\mu} \leq \omega_{i} \\
\frac{1}{\mu}-\omega_{i}, & \text { if } \frac{1}{\mu}>\omega_{i}
\end{array} .\right.
$$


We notice that, the solution of subproblem 2 presented in Eq. 25) can also be obtained from Eq. (23) for by replacing $b_{i}^{2}$ with 1 and $e_{i}^{2}$ with 0 . In the absence of eavesdropper, the solution of this simplified problem is the famous water-filling solution as given in Eq. (25), where the base level is $\omega_{i}$ and water level is the inverse of the dual variable $\frac{1}{\mu}$. When the security constraint is present, however, the problem takes on an interesting structure. In such case, we can still consider the water level as $\frac{1}{\mu}$. However, we will use $\frac{\omega_{\nu+i}}{b_{i}^{2}-e_{i}^{2}}$ as a base level to take into account the additional constraint due to secrecy. For the parallel channels $j=k-r+1, \ldots, k$, with components only towards Bob's direction, the base level will still be $\omega_{j}$ (since, $b_{j-\nu}^{2}=1$ and $e_{j-\nu}^{2}=0$ ). Similar to water-filling, we will allocate power only when the water level is above the base level. However, the power level in this case will not be the difference between the water level and base level, i.e. $\frac{1}{\mu}-\frac{\omega_{\nu+i}}{b_{i}^{2}-e_{i}^{2}}$. Instead, it will be a non-linear function of the difference as given in Eq. (24). As a result, the achievable secrecy rate can be written as

$$
R_{s}^{\mathrm{g}}=\sum_{i: b_{i}>e_{i}} \log \left(\frac{1+\frac{b_{i}^{2}}{\omega_{\nu+i}} p_{\nu+i}^{\mathrm{g}}}{1+\frac{e_{i}^{2}}{\omega_{\nu+i}} p_{\nu+i}^{\mathrm{g}}}\right)+\sum_{j=k-r+1}^{k} \log \left(1+\frac{1}{\omega_{j}} p_{j}^{\mathrm{g}}\right)
$$

\section{B. Low SNR Approximation}

1) Second order optimal signaling: For second order optimal signaling [?], the first and the second order derivatives of the mutual information achieved at zero SNR matches with those achieved using Gaussian input. In general, quadratic symmetric signaling such as QPSK or any other signaling distribution that can be written as a mixture of QPSK (i.e., $M$-QAM, for $M \geq 4$ ) are second order optimal. For second order optimal signaling, the low SNR approximation of MMSE (i.e., the first derivative of mutual information) is the same as that of Gaussian signaling. Hence, we can use the same water-filling power allocation solution as presented in Eq. (24), (25) in Section $\mathrm{V}-\mathrm{A}$.

2) Non-second order optimal signaling: 1-D signaling schemes such as BPSK and $M$-PAM are not second order optimal. A low SNR approximation of such signaling is given in [?] as

$$
\operatorname{mmse}(\rho)=1-2 \rho+o\left(\rho^{2}\right) \text {. }
$$

Based on the above equation, low SNR power allocation $\left\{p_{i}^{\text {low }}\right\}$ for non-second order optimal signaling are given below in two cases: 


$$
\begin{aligned}
& p_{\nu+i}^{\text {low }}=\left\{\begin{array}{ll}
0, & \text { when } \frac{1}{\mu} \leq \frac{\omega_{\nu+i}}{b_{i}^{2}-e_{i}^{2}} \\
\frac{\omega_{\nu+i}}{2} \mu\left(\frac{1}{\mu}-\frac{\omega_{\nu+i}}{b_{i}^{2}-e_{i}^{2}}\right) & \text { when } \frac{1}{\mu}>\frac{\omega_{\nu+i}}{b_{i}^{2}-e_{i}^{2}}
\end{array} \quad\left\{i: b_{i}>e_{i}\right\}\right. \\
& p_{i}^{\text {low }}=\left\{\begin{array}{ll}
0, & \text { when } \frac{1}{\mu} \leq \omega_{i} \\
\frac{\omega_{i}}{2} \mu\left(\frac{1}{\mu}-\omega_{i}\right), & \text { when } \frac{1}{\mu}>\omega_{i}
\end{array}, i=k-r+1, \ldots, k .\right.
\end{aligned}
$$

The achievable secrecy rate at low SNR can be approximated as follows

$$
R_{s}^{\text {low }}=\sum_{i: b_{i}>e_{i}}\left(b_{i}^{2}-e_{i}^{2}\right)\left(p_{\nu+i}^{\text {low }}-\left(p_{\nu+i}^{\text {low }}\right)^{2}\right)+\sum_{j=k-r+1}^{k}\left(p_{j}^{\text {low }}-\left(p_{j}^{\text {low }}\right)^{2}\right) .
$$

We notice that, similar to the case of Gaussian and second-order optimal signaling, we can use a water-filling strategy with water level $\frac{1}{\mu}$ and base level $\frac{\omega_{\nu+i}}{b_{i}^{2}-e_{i}^{2}}$ and assuming $b_{j-\nu}^{2}=1$ and $e_{j-\nu}^{2}=0$ for $j=k-r+1, \ldots, k$. The power level is still function of

$$
\frac{1}{\mu}-\frac{\omega_{\nu+i}}{b_{i}^{2}-e_{i}^{2}}
$$

which is the difference between water level and base level. The functions are now slightly different in (Eq. (28) and (29)).

\section{High SNR Approximation}

1) $\operatorname{rank}\left(\mathbf{H}_{e}\right)=m_{a}:$ In this case $\operatorname{dim}\left(S_{b}\right)=r=0$. For the power constraint in Eq. (12), we get the following complementary slackness condition

$$
\mu\left(\sum_{i: b_{i}>e_{i}} \omega_{\nu+i} p_{\nu+i}-P_{T}\right)=0
$$

Based on proposition 2, the optimal power $p^{*}$ for maximum secrecy satisfies $p^{*} \leq p^{\prime}$. Therefore, at very high SNR when $P_{T} \rightarrow \infty$, secrecy rate for finite-alphabet input is maximized by using a fraction of the total available power. The power constraint inequality becomes a strict inequality. In the above complementary slackness condition, we attain $\mu=0$. Denoting high SNR power allocation as $p_{i}^{\text {high }}$, we re-write Eq. (20) as

$$
b_{i}^{2} \operatorname{mmse}\left(b_{i}^{2} p_{\nu+i}^{\text {high }}\right)=e_{i}^{2} \operatorname{mmse}\left(e_{i}^{2} p_{\nu+i}^{\text {high }}\right) .
$$


In [?], based on the sub-optimum estimator $\hat{s}(y, \rho)=\arg \min _{s_{k}}\left|y-\sqrt{\rho} s_{k}\right|$ the following MMSE approximation at high SNR is found:

$$
\operatorname{mmse}(\rho) \approx K \exp \left\{-\frac{d^{2}}{4} \rho\right\},
$$

in which $K$ is a constant and $d$ is the minimum distance between two signaling points in the discrete unit variance input constellation.

Reference [?] also provided a table containing formula for calculating $d$ of different finitealphabet constellations. Using Eq. (33), we obtain the following high SNR approximation of power allocation

$$
p_{\nu+i}^{\text {high }}=\frac{\log \sigma_{i}^{2}}{\frac{d^{2}}{4} \frac{\left(b_{i}^{2}-e_{i}^{2}\right)}{\omega_{\nu+i}}}, \text { for } i \text { such that } b_{i}>e_{i} .
$$

In [?], it was observed that, at high SNR regime, the power allocation for parallel Gaussian channel with finite-alphabet demonstrates a channel inversion characteristic. In other words, stronger channels receive less power allocation. This is because the mutual information of a $M$ ary constellation cannot exceed $\log _{2} M$ bits/s/Hz, Thus, there is little incentive to allocate more power to a channel once the mutual information is near saturation. Instead, additional power is better allocated to weaker channels for higher rate. In Eq. (34), we observe a similar channel inversion phenomenon, although in this case, the effective channel $\frac{\left(b_{i}^{2}-e_{i}^{2}\right)}{\omega_{\nu+i}}$. This is in sharp contrast to both the water-filling power allocation at low SNR regime and the power allocation for Gaussian input (see Eq. (28)), where the power allocation was proportional to the effective channel.

2) $\operatorname{rank}\left(\mathbf{H}_{e}\right)<m_{a}$ : Based on Eq. (33), $\operatorname{mmse}(\rho)$ decays exponentially to zero as $\rho \rightarrow \infty$. From Eq. (18), therefore, we find that $\mu \rightarrow 0$ as $P_{T} \rightarrow \infty$. Hence, for the subset of parallel channels, $i: b_{i}>e_{i}$, Eq. (34) will still provide a high SNR approximation of power allocation.

The subset of parallel channels $j=k-r+1, \ldots, k$ are in the subspace $\mathcal{S}_{b}$. For these channels, there are no components in Eve's subspace and a similar channel inversion style power allocation can be achieved as presented in [?] with an effective channel $\frac{1}{\omega_{j}}$. For an $M$-ary constellation, mutual information for these channels will become close to $\log _{2} M$ at high SNR.

In summary, for the subset of parallel channels $i: b_{i}>e_{i}$, a channel inversion based power allocation based on the effective channel $\frac{b_{i}^{2}-e_{i}^{2}}{\omega_{\nu+i}}$ will be performed, whereas for the set of parallel channels $j=k-r+1, \ldots, k$, a channel inversion type power allocation based on 
effective channel gains $1 / \omega_{j}$ will be performed. A high SNR approximation of the achievable secrecy rate for the case $\operatorname{rank}\left(\mathbf{H}_{e}\right)<m_{a}$ is given by

$$
R_{s}^{\text {high }} \approx r \log M+\sum_{i: b_{i}>e_{i}}\left[\mathcal{I}\left(\frac{b_{i}^{2}}{\omega_{\nu+i}} p_{\nu+i}^{\text {high }}\right)-\mathcal{I}\left(\frac{e_{i}^{2}}{\omega_{\nu+i}} p_{\nu+i}^{\text {high }}\right)\right] .
$$

\section{NUMERICAL RESULTS}

\section{A. Transmitter with Accurate Eavesdropper CSI}

Without any loss of generality, we assume equal noise power level at receivers of both Bob and Eve. We also assume that Alice has full CSI of both Bob and Eve. Our numerical results average over 500 channel realization, where each entry of both Bob's and Eve's channel matrices is i.i.d. complex random Gaussian variable with zero mean and unit variance.

In Fig. 2, we present numerical test results for a $5 \times 5 \times 5$ MIMOME system. In this test, we ensure that each realization of $\mathbf{H}_{e}$ is non-singular, i.e., $\operatorname{rank}\left(\mathbf{H}_{e}\right)=5=m_{a}$. Since, $\operatorname{rank}\left(\mathbf{H}_{e}\right)=$ $m_{a}$, there is no parallel channel only directed at Bob. In addition to result for the proposed power allocation (PA) algorithm, we also present results obtained using the water-filling PA of Section $\mathrm{V}-\mathrm{A}$ and results from equal power over all channels (uniform PA) [?]. We also present high SNR approximation results for every tested constellation as well as the low SNR approximation result for BPSK. For other constellations (QPSK, 16-QAM and 64-QAM), low SNR approximation gives the same result as the water-filling PA.

As seen in Fig. 2, at high SNR, power allocations according to water-filling and uniform strategies would drop the secrecy rate to almost zero. This result is intuitive. For finite-alphabet, the achievable mutual information at high power approaches the saturation value of $\log _{2} M$. For Gaussian input, however, the mutual information or the capacity increases monotonically with increasing power. Since both water-filling and uniform strategies assume a Gaussian input distribution, at high SNR both schemes would use more power to transmit signals. This strategy drops the secrecy rate asymptotically to zero as the difference in mutual information between Alice-to-Bob and Alice-to-Eve narrows with at very high SNR. Fig. 2 also indicates that the high SNR approximation analysis closely matches the secrecy rate at high SNR regime. Similarly, the secrecy rate at low SNR is also closely approximated by the low-SNR approximation analytical result.

Fig. 3 presents test results for a $5 \times 5 \times 3$ MIMOME system. Because in this case $\operatorname{rank}\left(\mathbf{H}_{e}\right)<$ $m_{a}$, there are parallel channel components only directed towards Bob. Therefore, results obtained 
in this case are different from Fig. 2. Specifically, we observe that, even though secrecy rates using water-filling and uniform PA schemes at high SNR drops from the maximum attainable value, they do not approaches zero as in Fig. 2. The reason is due to the existence of channels directed only at Bob. For the parallel channels with components along both at Bob's and Eve's direction, the difference in mutual information between Alice-to-Bob and Alice-to-Eve narrows at high SNR. However, for each of the parallel channels with components only in Bob's direction, the secrecy rate would approach $\log _{2} M$. Therefore, at high SNR the total secrecy rate approaches $r \log _{2} M$. Moreover, our proposed PA algorithm can achieve some additional non-zero secrecy rate from the channels directed at both Bob and Eve, in addition to those parallel channels only in Bob's direction.

Both Fig. 2 and 3 indicate that when using Gaussian assumption for finite-alphabet inputs, there is a threhold $S N R$ above which the achievable secrecy rate starting to decrease. The value of this threshold SNR is higher for the higher order constellation. Therefore, by adaptively switching to the next higher order modulation format once we reach the threshold point for a particular constellation, we will be able to use Gaussian water-filling PA algorithm even for finite-alphabet inputs. We also notice similar threshold SNR for uniform PA. In addition, uniform PA also achieves secrecy rate close to the water-filling PA. Therefore, a very simple but near optimal strategy would be to use uniform power allocation and start switching to the next higher order modulation once above each threshold SNR.

\section{B. Transmitter with Partial Eavesdropper CSI}

Thus far, our analysis assumes that Alice possesses full channel information of Eve. In practice, however, Eve's CSI or even the presence of a passive Eve is difficult to determine. Therefore, in this section, we will numerically evaluate the scenario when Alice only has access to partial (statistical) information regarding Eve's channel state. In particular, let Eve's channel consists of

$$
\mathbf{H}_{e}=\hat{\mathbf{H}}_{e}+\mathbf{E}_{e}
$$

Here, $\hat{\mathbf{H}}_{e}$ is Eve's mean CSI known to Alice whereas $\mathbf{E}_{e}$ is the CSI uncertainty which is modeled as zero mean white Gaussian noise with variance $\sigma_{e}^{2}$, i.e., $\mathbf{E}_{e} \sim \mathcal{C N}\left(\mathbf{0}, \sigma_{e}^{2} \mathbf{I}\right)$.

In this case, if Alice performs power allocation based on the known observation $\hat{\mathbf{H}}_{e}$ by disregarding the uncertainty, the power allocation may not be optimal. In fact, Alice may even 
lose secrecy. In the following proposition, we present the achievable ergodic secrecy rate for a given power allocation $\mathbf{p}$.

Proposition 4: For a given power allocation $\mathbf{p}$ based on the known observation $\hat{\mathbf{H}}_{e}$ without considering the uncertainty, the achievable ergodic secrecy rate $R_{\mathrm{sec}}(\mathbf{p})$ can be given as follows

$$
\begin{aligned}
R_{\mathrm{sec}}(\mathbf{p})=\sum_{i: b_{i}>e_{i}}\left[\mathcal{I}\left(\frac{b_{i}^{2}}{\omega_{\nu+i}} p_{\nu+i}\right)-\right. & \left.\mathbb{E}\left\{\mathcal{I}\left(\frac{\left(e_{i}^{2}+\tilde{e}_{\nu+i}\right)^{2}}{\sigma_{\nu+i} \omega_{\nu+i}} p_{\nu+i}\right)\right\}\right] \\
& +\sum_{j=k-r+1}^{k}\left[\mathcal{I}\left(\frac{1}{\omega_{j}} p_{j}\right)-\mathbb{E}\left\{\mathcal{I}\left(\frac{\tilde{e}_{j}^{2}}{\sigma_{j} \omega_{j}} p_{j}\right)\right\}\right]
\end{aligned}
$$

Here, $\tilde{e}_{i} \sim \mathcal{C N}\left(0, \sigma_{e}^{2} \omega_{i}\right), i=1, \ldots, k$ are i.i.d. random. Also, $\sigma_{i}^{2}=1+\sigma_{e}^{2}\left(\sum_{\ell=1}^{k} p_{\ell}-p_{i}\right)$.

Proof: See Appendix D.

From Eq. (36) we notice that, data symbols $s_{k-r+1}, \ldots, s_{k}$ - previously only observed by Bob, are also seen by Eve now. In addition, for the symbols $s_{\nu+i}, \ldots, s_{\nu+s}$ - observed by both Bob and Eve, an additional uncertainty term $\tilde{e}_{\nu+i}$ is added to the mutual information of Eve.

In Fig. 4 and 5, we present test results of achievable ergodic secrecy rate for different values of variance of channel uncertainty, for $5 \times 5 \times 5$ and $5 \times 5 \times 3$ MISOME systems, respectively. Here, Alice is only aware of the mean CSI $\hat{\mathbf{H}}_{e}$ and use this CSI for power control without considering the uncertainty component $\mathbf{E}_{e}$. We present test results for different values of available transmission power. Both Fig. 4 and 5 indicate that the achievable ergodic secrecy rate decreases with larger channel uncertainty. In addition, we observe from Fig. 4 that the achievable ergodic secrecy rate plots for transmission power $P_{T} \geq 5$ stays the same. This is because Alice does not require all available power to transmit at higher SNR. Hence, the power allocation stays the same at higher SNR. However, in Fig. 5, we observe that the achievable ergodic secrecy rate in fact decreases with larger transmission power for $P_{T}>10 \mathrm{~dB}$. In this case, rank $\left(\hat{\mathbf{H}}_{e}\right)<m_{a}$. When Alice only uses this information for power control without considering the uncertainty, Alice will likely allocate more power to the bank of parallel channels which only has components toward Bob. At high SNR, Alice will allocate more powers to these channels. However, as shown in Eq. (36), because of channel uncertainty $\mathbf{E}_{e}$, Eve now also possesses components along these channels. In other words, Eve can also receive signals from these channels. As SNR grows large, the mutual information for finite alphabet saturates to $\log _{2} M$. As a result, Eve can receive nearly full data information in these channel and consequently, the achievable ergodic secrecy rate will 
decrease at high SNR.

We note that, in order to maximize the achievable ergodic secrecy rate under channel uncertainty, one needs to solve the following optimization problem

$$
\begin{aligned}
\underset{\mathbf{p}}{\operatorname{maximize}} \sum_{i=1}^{s}\left[\mathcal{I}\left(\frac{b_{i}^{2}}{\omega_{\nu+i}} p_{\nu+i}\right)-\mathbb{E}\left\{\mathcal{I}\left(\frac{\left(e_{i}^{2}+\tilde{e}_{\nu+i}\right)^{2}}{\sigma_{\nu+i} \omega_{\nu+i}} p_{\nu+i}\right)\right\}\right] \\
+\sum_{j=k-r+1}^{k}\left[\mathcal{I}\left(\frac{1}{\omega_{j}} p_{j}\right)-\mathbb{E}\left\{\mathcal{I}\left(\frac{\tilde{e}_{j}^{2}}{\sigma_{j} \omega_{j}} p_{j}\right)\right\}\right]
\end{aligned}
$$

subject to: $\quad \sum_{i=1}^{s} p_{\nu+i}+\sum_{j=k-r+1}^{k} p_{j} \leq P_{T}$.

Investigation of this optimization problem is beyond the scope of this work. However, in [?], a power allocation algorithm to maximize the achievable ergodic secrecy rate under arbitrary input distribution is provided for a multiple-input single-output and single-eavesdropper scenario. An extension of the power allocation algorithm provided in [?] can also be used to solve the above optimization problem.

\section{CONCLUSION}

This work considers the effect of practical finite-alphabet inputs on the secrecy performance of an MIMOME system. Our investigation led to the application of a precoding matrix to convert the MIMOME system into a bank of parallel channels so as to reformulate the achievable secrecy rate problem. We proposed a decentralized dual decomposition and a corresponding power allocation algorithm to maximize the achievable secrecy rate based on the proposed

precoding for channel transformation. We analyzed the Gaussian input as a special case and provided a water-filling inspired power allocation strategy. Furthermore, we derived analytical results of achievable secrecy rate based on approximations at low and high SNR scenarios. Our results show that power allocation strategy based on Gaussian input is far from optimal when applied blindly in finite-alphabet input situation and may in fact be very risky by driving the secrecy rate to zero at higher SNR.

\section{APPENDIX A}

\section{ProOF OF PROPOSITION 1}

An achievable secrecy rate for an arbitrary distribution of input $\mathrm{x}$ can be obtained by solving the following optimization problem 


$$
\begin{aligned}
& \underset{\mathbf{K}_{\mathbf{x}}}{\operatorname{maximize}} I\left(\mathbf{x} ; \mathbf{y}_{b}\right)-I\left(\mathbf{x} ; \mathbf{y}_{e}\right) \\
& \text { subject to : } \quad \mathbf{K}_{\mathbf{x}} \succeq 0, \mathbf{K}_{\mathbf{x}}=\mathbf{K}_{\mathbf{x}}^{\mathbf{H}} \\
& \\
& \operatorname{tr}\left(\mathbf{K}_{\mathbf{x}}\right) \leq P_{T}
\end{aligned}
$$

When we apply the precoding matrix $\mathbf{W}$ from Eq. (4), the input of the system becomes $\mathbf{x}=$ Ws. The objective function of the above optimization problem can be written as follows

$$
\begin{aligned}
& I\left(\mathbf{x} ; \mathbf{y}_{b}\right)-I\left(\mathbf{x} ; \mathbf{y}_{e}\right) \\
= & I\left(\mathbf{s} ; \mathbf{H}_{b} \mathbf{W} \mathbf{s}+\mathbf{n}_{b}\right)-I\left(\mathbf{s} ; \mathbf{H}_{e} \mathbf{W} \mathbf{s}+\mathbf{n}_{e}\right) \\
= & I\left(\mathbf{s} ; \tilde{\mathbf{H}}_{b} \mathbf{s}+\tilde{\mathbf{n}}_{b}\right)-I\left(\mathbf{s} ; \tilde{\mathbf{H}}_{e} \mathbf{s}+\tilde{\mathbf{n}}_{e}\right) \\
= & {\left[\sum_{i=1}^{s} \mathcal{I}\left(b_{i}^{2} p_{k-r-s+i}\right)+\sum_{j=k-r+1}^{k} \mathcal{I}\left(p_{j}\right)\right]-\left[\sum_{\ell=1}^{k-r-s} \mathcal{I}\left(p_{\ell}\right)+\sum_{i=1}^{s} \mathcal{I}\left(e_{i}^{2} p_{k-r-s+i}\right)\right] } \\
= & \sum_{j=k-r+1}^{k} \mathcal{I}\left(p_{j}\right)+\sum_{i=1}^{s}\left[\mathcal{I}\left(b_{i}^{2} p_{k-r-s+i}\right)-\mathcal{I}\left(e_{i}^{2} p_{k-r-s+i}\right)\right]-\sum_{\ell=1}^{k-r-s} \mathcal{I}\left(p_{\ell}\right) .
\end{aligned}
$$

Note that (40) follows from (39) since linear unitary transformation of channel outputs preserves mutual information. Because (40) represents the difference in mutual information between a subset of parallel channels, we can rewrite (40) into the summation form of (41).

Since $\mathbf{s}$ is a random vector with identity correlation matrix, $\mathbf{K}_{\mathbf{x}}=\mathbb{E}\left[\mathbf{x x}^{H}\right]=\mathbf{W W}^{H}$, we have $\operatorname{tr}\left(\mathbf{K}_{\mathbf{x}}\right)=\sum_{i=1}^{k} \omega_{i} p_{i}$. Therefore, we can reformulate the optimization problem in (37) as follows

$$
\begin{aligned}
& \underset{\left\{p_{i}\right\}}{\operatorname{maximize}} \sum_{i=1}^{s}\left[\mathcal{I}\left(b_{i}^{2} p_{k-r-s+i}\right)-\mathcal{I}\left(e_{i}^{2} p_{k-r-s+i}\right)\right]-\sum_{i=1}^{k-r-s} \mathcal{I}\left(p_{i}\right)+\sum_{i=k-r+1}^{k} \mathcal{I}\left(p_{i}\right) \\
& \text { subject to : } \quad \sum_{i=1}^{k} \omega_{i} p_{i} \leq P_{T}
\end{aligned}
$$

Note that the above optimization problem is a distributed power allocation problem for the bank of parallel channels shown in Fig. 1, where the variables $\left\{p_{i}\right\}$ 's are coupled through the total power constraint. Because $\mathcal{I}() \geq$.0 , we have optimum power allocation $p_{i}^{*}=0$ for $i=1, \ldots, k-r-s$. In addition, since $\mathcal{I}(\gamma)$ are monotonically increasing in $\gamma, \mathcal{I}\left(b_{i}^{2} p_{k-r-s+i}\right)-$ 
$\mathcal{I}\left(e_{i}^{2} p_{k-r-s+i}\right) \leq 0$ whenever $b_{i} \leq e_{i}$, hence $p_{i}^{*}=0$, for all $i$ with $b_{i} \leq e_{i}$. Furthermore, from Fig. 1, we observe that symbols $s_{k+1}, \ldots, s_{m_{a}}$ are transmitted towards the direction of $\mathcal{S}_{n}$ and is not part of the optimization problem (43). Hence, it will be wasteful to expend any transmission power to transmit these symbols. Therefore, $p_{i}^{*}=0$ for $i=k+1, \ldots, m_{a}$. By change of variables, $p^{\prime}=\omega_{i} p_{i}$, and then replacing $p^{\prime}$ with $p$, we obtain the optimization problem in Eq. (12).

\section{APPENDIX B}

\section{PROOF OF PROPOSITION 2}

If $b_{i}^{2} \leq e_{i}^{2}$, the difference in mutual information between Alice-to-Bob and Alice-to-Eve channel will be negative. Hence, no secrecy is possible. As a result, the optimal power allocation should be zero.

When $b_{i}^{2}>e_{i}^{2}$, it is possible to achieve a positive secrecy rate. If, in the solution of the original problem (12), the sum power constraint inequality becomes a strict inequality, then $\mu=0$ according to the KKT condition [?]. Such condition can occur at high SNR regime. Therefore, the solution of problem (20) will be achieved by the power allocation $p^{\prime}$ that renders the MMSE difference function in Eq. (21) zero. If the MMSE difference function admits a unique zero solution, then the solution of Eq. (20) will be optimal, i.e., $p_{\nu+i}^{*}=p^{\prime}$.

If on the other hand, the sum power constraint in the original problem admits an equality, then $\mu>0$. If the MMSE difference function is monotonically decreasing for $0 \leq p \leq p^{\prime}$, then the function $f_{\mathrm{mmseD}}\left(p, b_{i}, e_{i}, \omega_{\nu+i}\right)-\mu$ will be zero for a power allocation value $p^{*}$ that is less than $p^{\prime}$. Now, if MMSE difference function is strictly monotonically decreasing, then the power allocation solution will be unique. Therefore, the solution of Eq. (20) will be optimal.

\section{APPENDIX C \\ ProOF OF PROPOSITION 3}

Define, $\rho_{1}=b_{i} p$ and $\rho_{2}=e_{i} p$. First, we will prove that the difference function, $g_{\mathrm{D}}\left(\rho_{1}, \rho_{2}\right)=$ $g\left(\rho_{1}\right)-g\left(\rho_{2}\right)$ admits a unique zero for $p>0$.

Assume that the strictly unimodal function $g(\rho)$ is strictly monotonically increasing for $\rho \leq m$ and strictly monotonically decreasing for $\rho>m$. When, $\rho_{2}<\rho_{1} \leq m$, the difference function $g_{\mathrm{D}}\left(\rho_{1}, \rho_{2}\right)$ cannot be zero due to the strictly monotonically increasing nature of the function $g(\rho)$. Similarly, when $\rho_{1}>\rho_{2}>m$, the difference function $g_{\mathrm{D}}\left(\rho_{1}, \rho_{2}\right)$ cannot be zero due to the 
strictly monotonically decreasing nature of the function $g(\rho)$. Therefore, the difference function $g_{\mathrm{D}}\left(\rho_{1}, \rho_{2}\right)$ can be zero only when $\rho_{2}<m<\rho_{1}$.

Suppose, for $p=p^{\prime}$, the difference function $g_{\mathrm{D}}\left(b_{i} p, e_{i} p^{\prime}\right)$ equals zero. For $p>p^{\prime}, g\left(b_{i} p\right)>$ $g\left(b_{i} p^{\prime}\right)$ and $g\left(e_{i} p\right)<g\left(e_{i} p^{\prime}\right)$ due to the strictly unimodal properties of $g(\rho)$. Hence, when $\rho_{2}<$ $m<\rho_{1}$, the difference function $g_{\mathrm{D}}\left(\rho_{1}, \rho_{2}\right)=g\left(\rho_{1}\right)-g\left(\rho_{2}\right)$ cannot be zero for $p>p^{\prime}$. The same argument holds for any point $p<p^{\prime}$. Therefore, when $b_{i}>e_{i}$, the difference function $g_{\mathrm{D}}\left(\rho_{1}, \rho_{2}\right)$ admits a unique zero for $p>0$. Hence the MMSE difference function $f_{\mathrm{mmseD}}\left(p, b_{i}, e_{i}, \omega_{\nu+i}\right)$ has a unique zero solution for $p>0$

\section{APPENDIX D}

\section{PROOF OF PROPOSITION4}

With $\mathbf{H}_{e}=\hat{\mathbf{H}}_{e}+\mathbf{E}_{e}$, Eq. (9) can be written as

$$
\tilde{\mathbf{y}}_{e}=\tilde{\mathbf{H}}_{e} \mathbf{s}+\boldsymbol{\Psi}_{e}^{H} \mathbf{E}_{e} \mathbf{W} \mathbf{s}+\tilde{\mathbf{n}}_{e}
$$

Here, $\tilde{\mathbf{H}}_{e}=\boldsymbol{\Psi}_{e}^{H} \hat{\mathbf{H}}_{e} \mathbf{W}=\boldsymbol{\Sigma}_{e}\left[\begin{array}{ll}\mathbf{I} & \mathbf{0}\end{array}\right] \mathbf{P}^{1 / 2}$. Now, the second term in the above equation can be expressed as $\boldsymbol{\Psi}_{e}^{H} \mathbf{E}_{e} \mathbf{W} \mathbf{s}=\tilde{\mathbf{E}}_{e} \mathbf{P}^{1 / 2} \mathbf{s}$, where, $\tilde{\mathbf{E}}_{e}=\hat{\mathbf{E}}_{e} \mathbf{B}$ and $\hat{\mathbf{E}}_{e}=\boldsymbol{\Psi}_{e}^{H} \mathbf{E}_{e} \mathbf{\Psi}_{a}$. We can decompose $\tilde{\mathbf{E}}_{e}$ as a sum of two matrices, where the first matrix is a diagonal matrix containing the diagonal elements of $\tilde{\mathbf{E}}_{e}$. The second matrix contains the non-diagonal entries of $\tilde{\mathbf{E}}_{e}$ and contains all zero elements in its diagonal. The decomposition is given below

$$
\tilde{\mathbf{E}}_{e}=\left[\begin{array}{ccccccc}
\tilde{e}_{1} & 0 & \ldots & 0 & 0 & \ldots & 0 \\
0 & \tilde{e}_{2} & \ddots & 0 & 0 & \ddots & 0 \\
\vdots & \vdots & \ddots & \vdots & \vdots & \ddots & \vdots \\
0 & 0 & \ldots & \tilde{e}_{k} & 0 & \ldots & 0 \\
0 & 0 & \ldots & 0 & 0 & \ldots & 0 \\
\vdots & \vdots & \ddots & \vdots & \vdots & \ddots & \vdots \\
0 & 0 & \ldots & 0 & 0 & \ldots & 0
\end{array}\right]+\left[\begin{array}{ccccccc}
0 & \tilde{e}_{12} & \ldots & \tilde{e}_{1 k} & 0 & \ldots & 0 \\
\tilde{e}_{21} & 0 & \ddots & \tilde{e}_{2 k} & 0 & \ddots & 0 \\
\vdots & \vdots & & \vdots & \ddots & \vdots & \\
\tilde{e}_{k 1} & \tilde{e}_{k 2} & \ldots & 0 & 0 & \ldots & 0 \\
\tilde{e}_{(k+1) 1} & \tilde{e}_{(k+1) 2} & \ldots & \tilde{e}_{(k+1) k} & 0 & \ldots & 0 \\
\vdots & \vdots & \ddots & \vdots & \vdots & \ddots & \vdots \\
\tilde{e}_{m_{e} 1} & \tilde{e}_{m_{e} 2} & \ldots & \tilde{e}_{m_{e} k} & 0 & \ldots & 0
\end{array}\right]
$$

Here, $\tilde{e}_{i j} \sim \mathcal{C N}\left(0, \sigma_{e}^{2} \omega_{j}\right)$. Based on the above decomposition and replacing $p_{i}$ with $\frac{p_{i}}{\omega_{i}}$, the $i$-th entry of the vector $\tilde{\mathbf{y}}_{e}$ can be expressed as follows

$$
\tilde{y}_{e_{i}}=\left(e_{i}^{\prime}+\tilde{e}_{i}\right) \frac{1}{\sqrt{\omega_{i}}} \sqrt{p_{i}} s_{i}+g_{i}+n_{e_{i}}
$$


Here, $g_{i}=\sum_{\ell=1, \ell \neq i}^{k} \tilde{e}_{i \ell} \frac{1}{\sqrt{\omega_{\ell}}} \sqrt{p_{\ell}} s_{\ell}$ and $e_{i}^{\prime}$ can be expressed as

$$
e_{i}^{\prime}= \begin{cases}1, & i=1, \ldots, k-r-s \\ e_{i-\nu}, & i=\nu+1, \ldots, \nu+s \text { and } \nu=k-r-s \\ 0, & i=k-r+1, \ldots, m_{e}\end{cases}
$$

Assume that the eavesdropper is performing conventional decoding on each of these parallel branch by considering the cross terms as a part of noise. Then the achievable ergodic secrecy rate for a given power allocation $\mathbf{p}$ can be written as in Eq. (36). If in case, Eve employs advanced decoding scheme, e.g. successive interference cancellation etc. then Eq. (36) will serve as a upper bound on the achievable ergodic secrecy rate. 


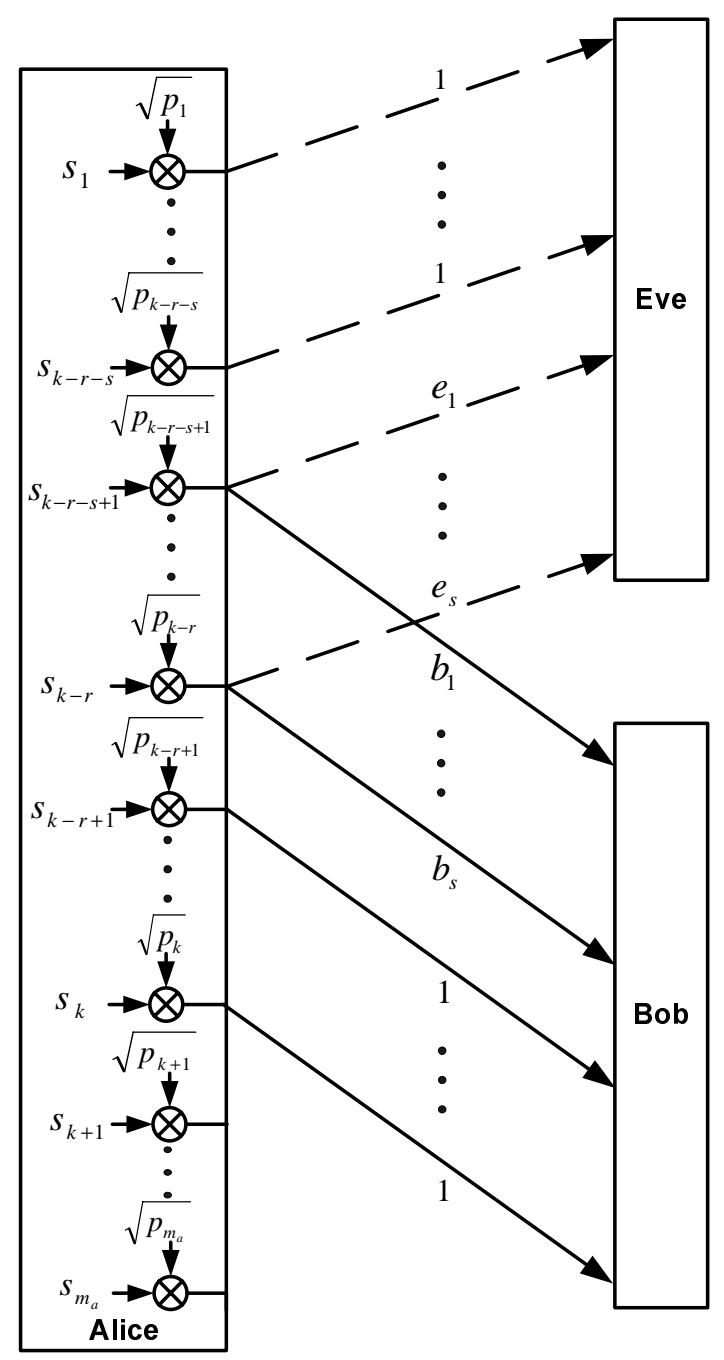

Fig. 1. Precoding matrix $\mathbf{W}=\mathbf{\Psi}_{a} \mathbf{B} \mathbf{P}^{1 / 2}$ converts MIMOME channel to a bank of parallel channels 


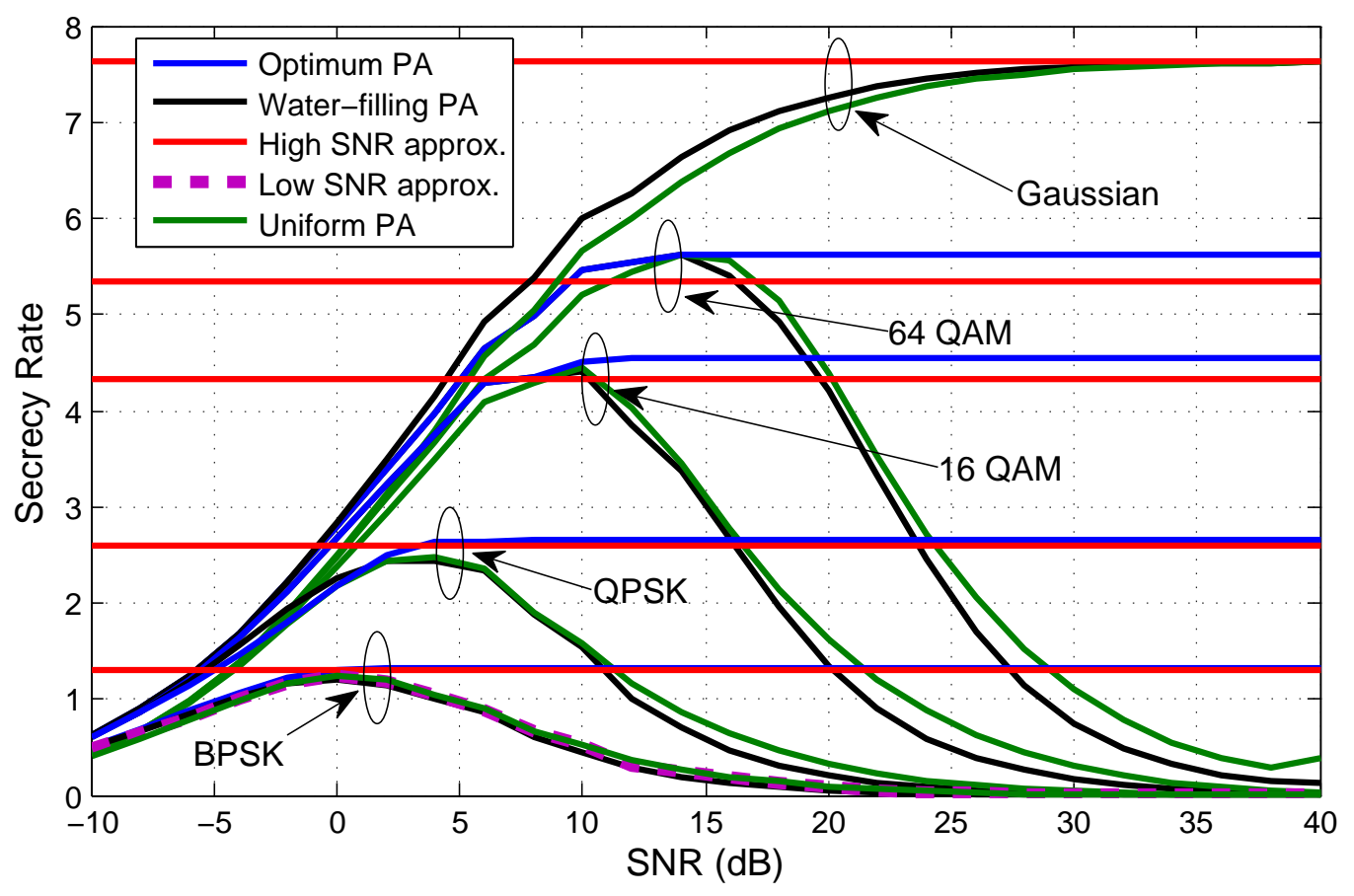

Fig. $2 . \quad 5 \times 5 \times 5$ MIMOME system

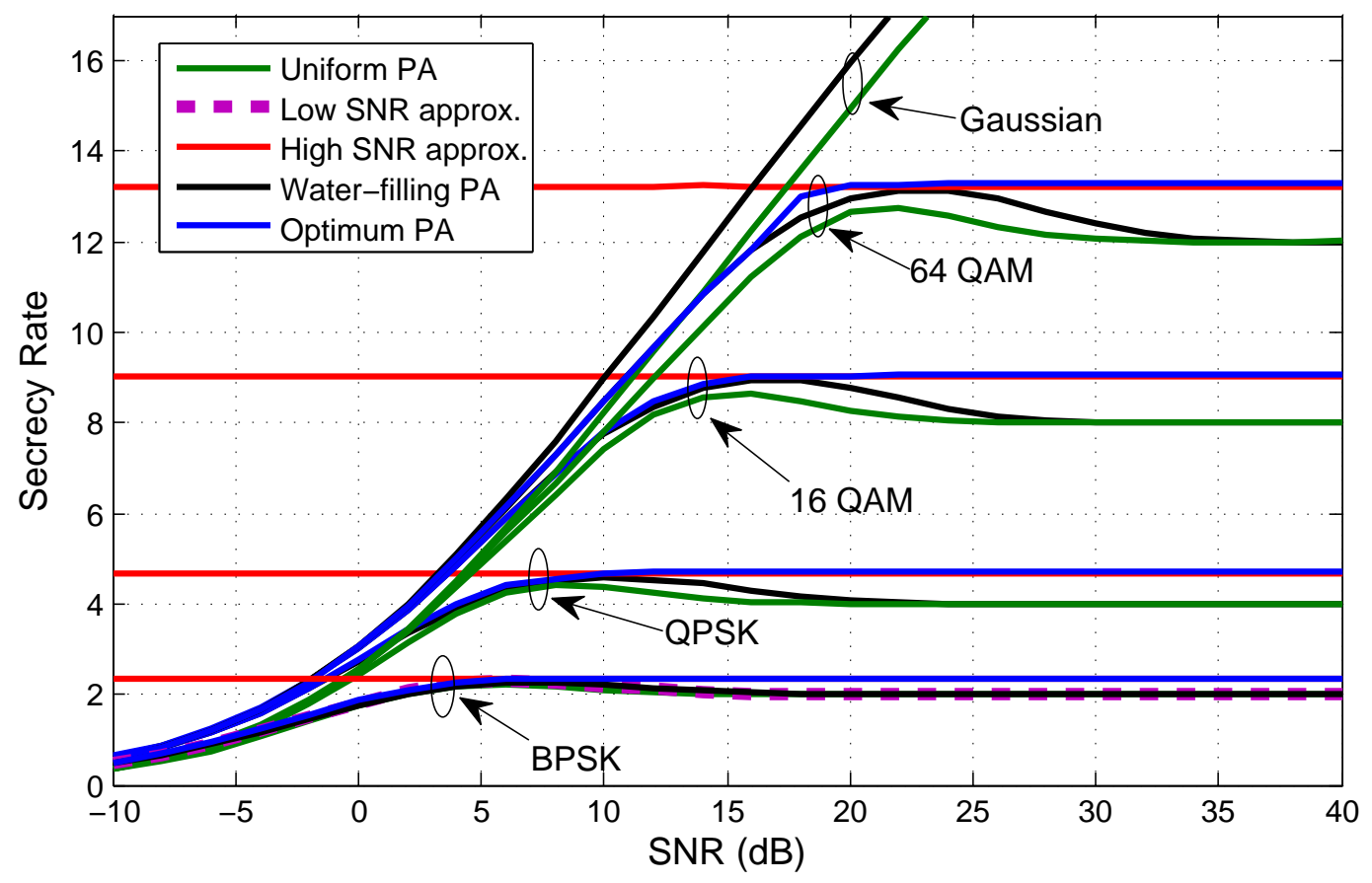

Fig. 3. $5 \times 5 \times 3$ MIMOME system 


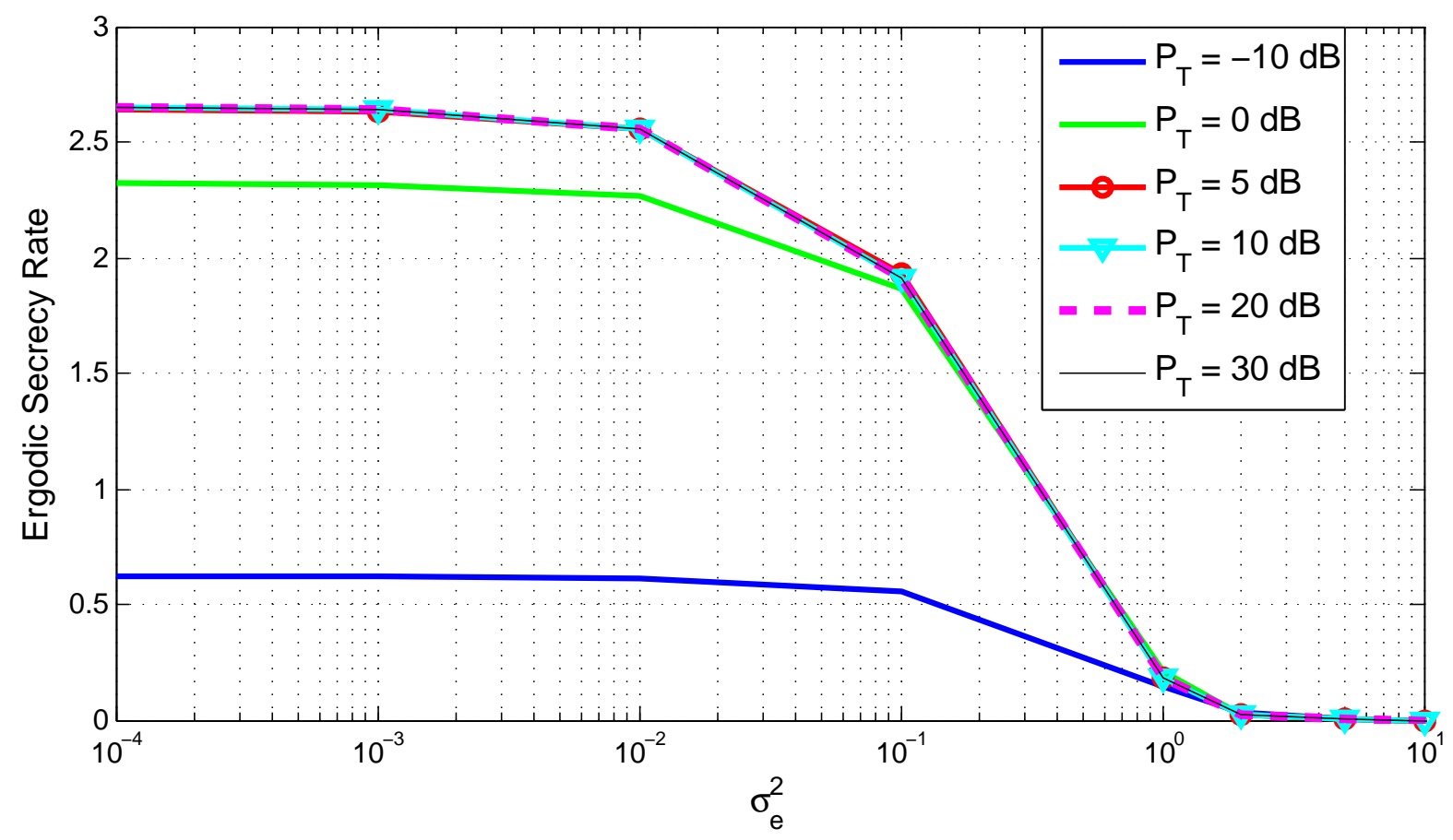

Fig. 4. Secrecy Rate of a $5 \times 5 \times 5$ MIMOME system with partial Eve's CSI

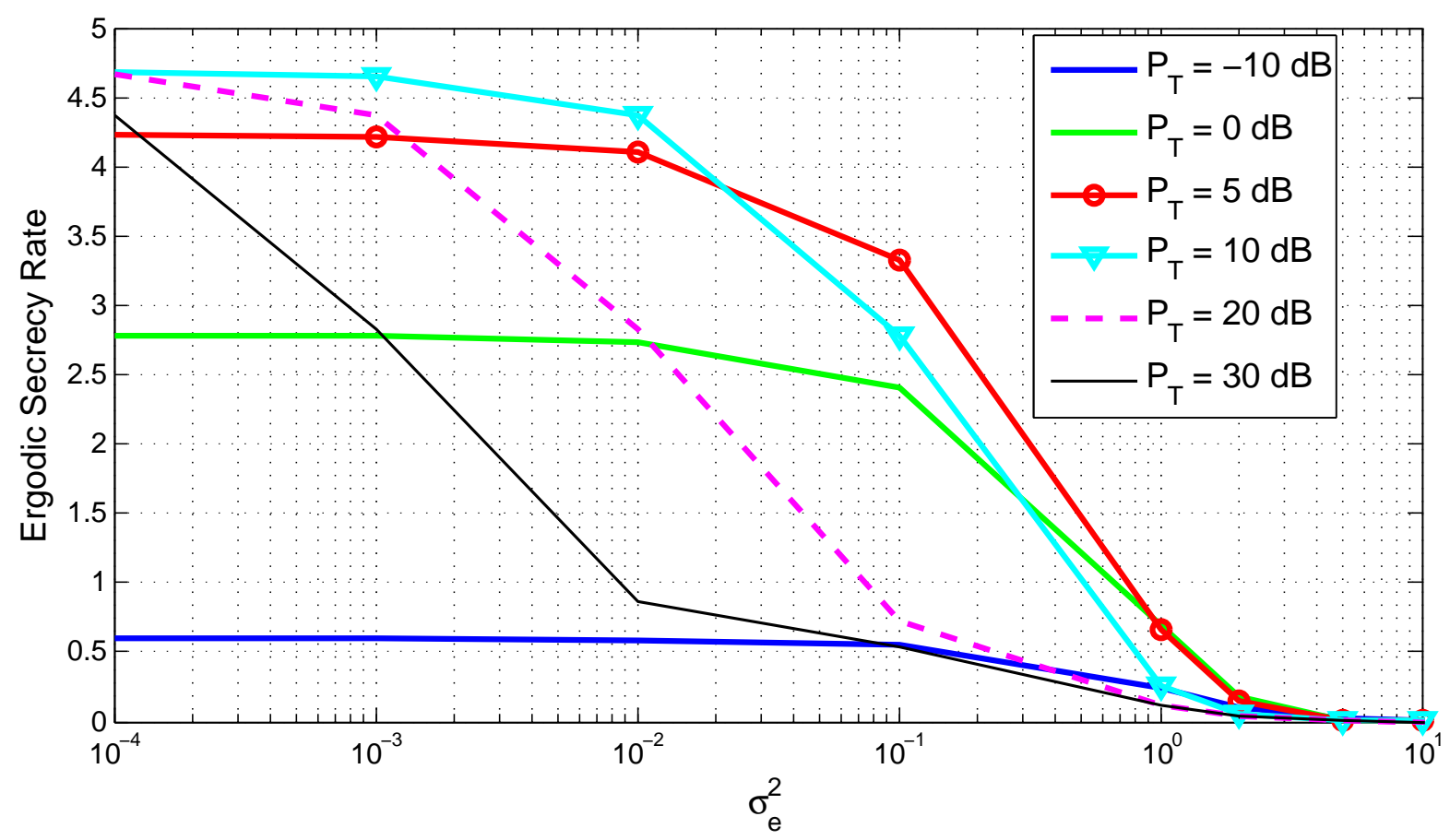

Fig. 5. Secrecy Rate of a $5 \times 5 \times 3$ MIMOME system with partial Eve's CSI 


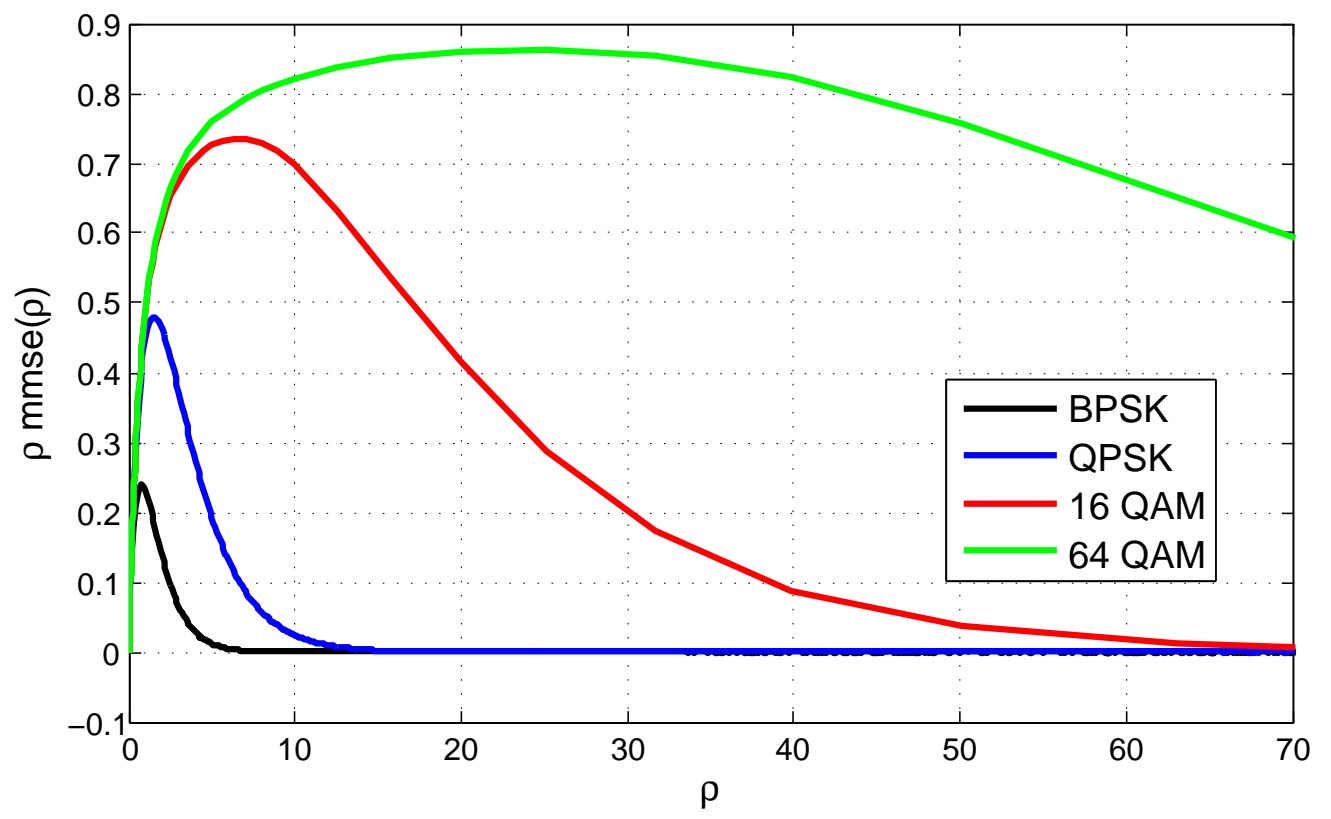

Fig. 6. $\rho$ vs, $\rho$ mmse $(\rho)$ plot for BPSK, QPSK, 16-QAM and 64-QAM constellations. 


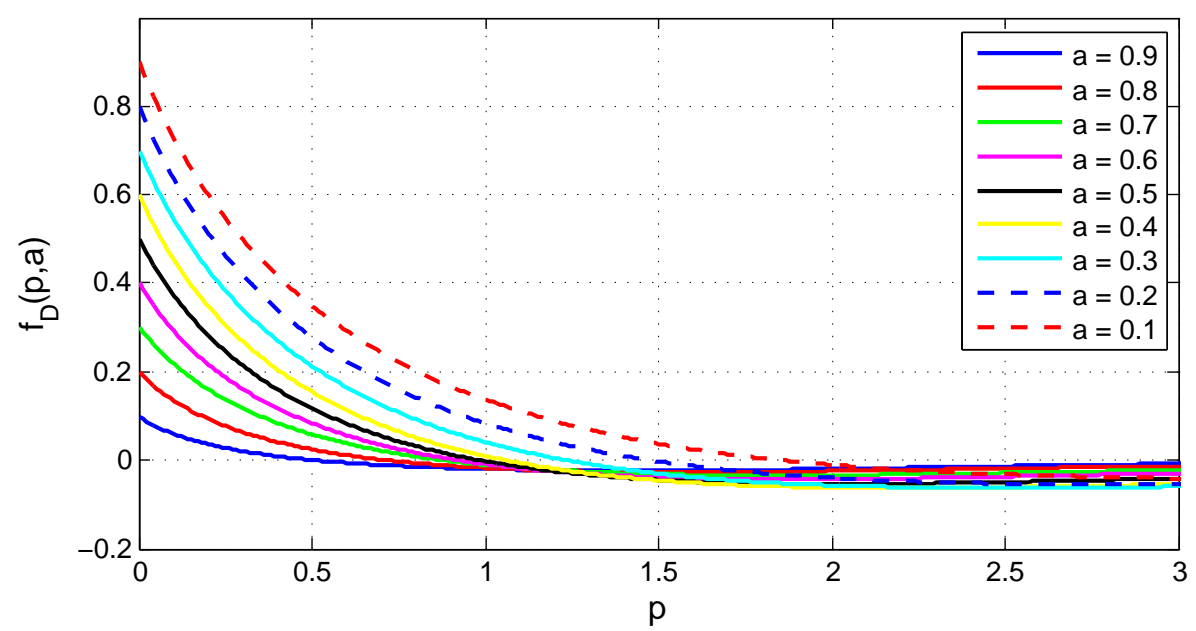

(a)

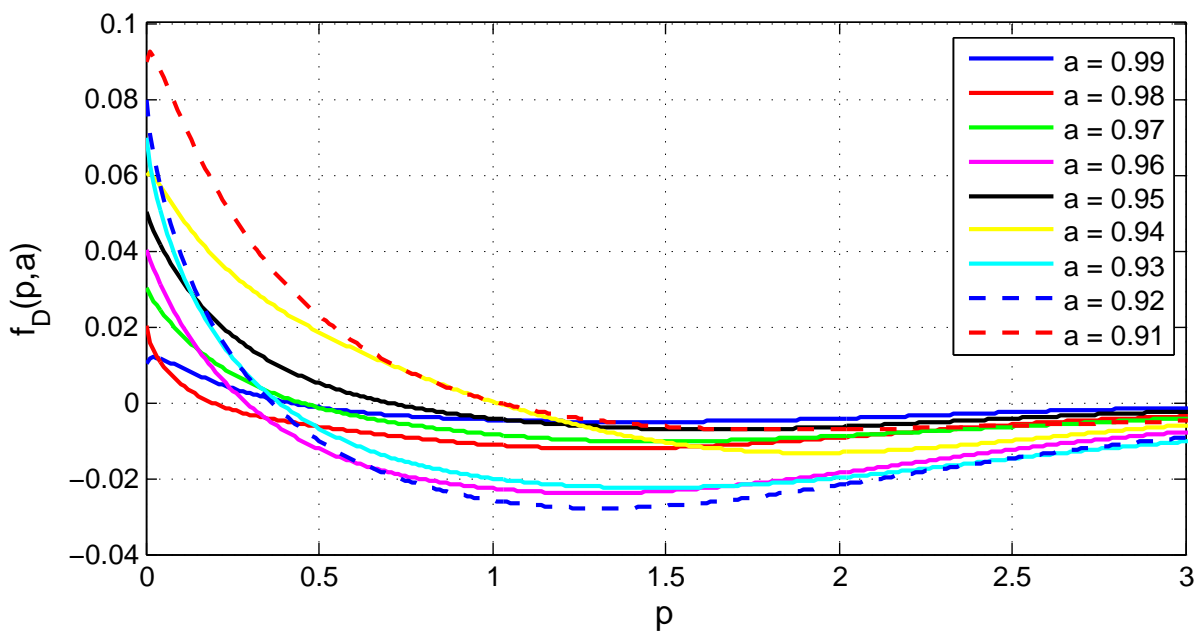

(b)

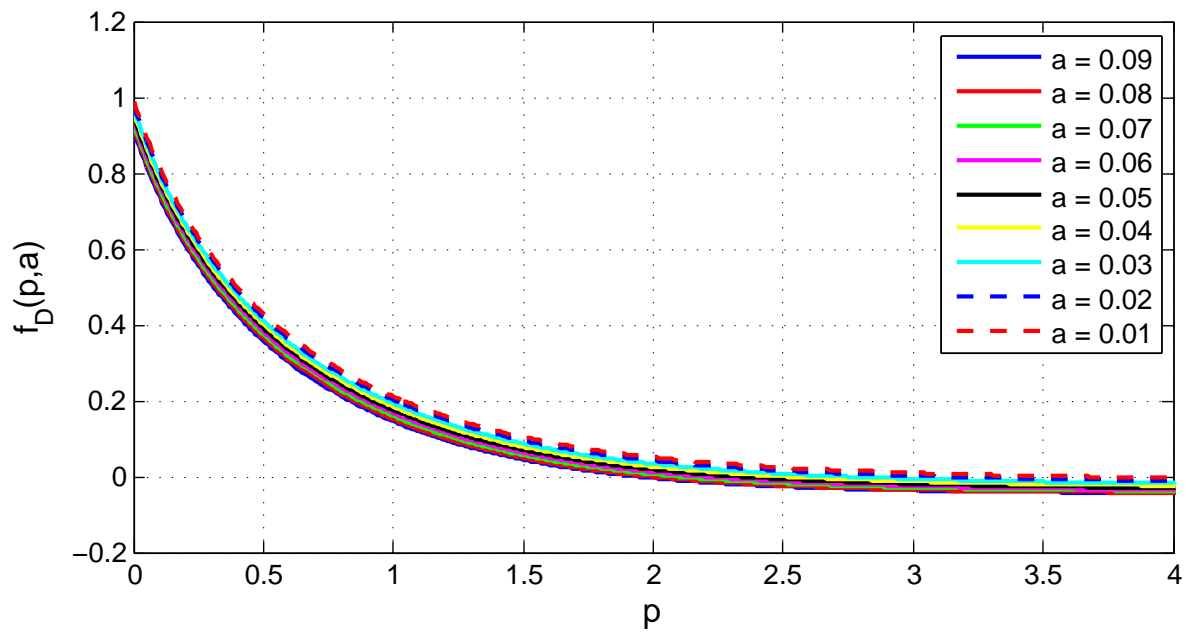

(c)

Fig. 7. MMSE difference function $f_{D}(p, a)$ for different values of $a=e_{i}^{2} / b_{i}^{2}$ for BPSK 


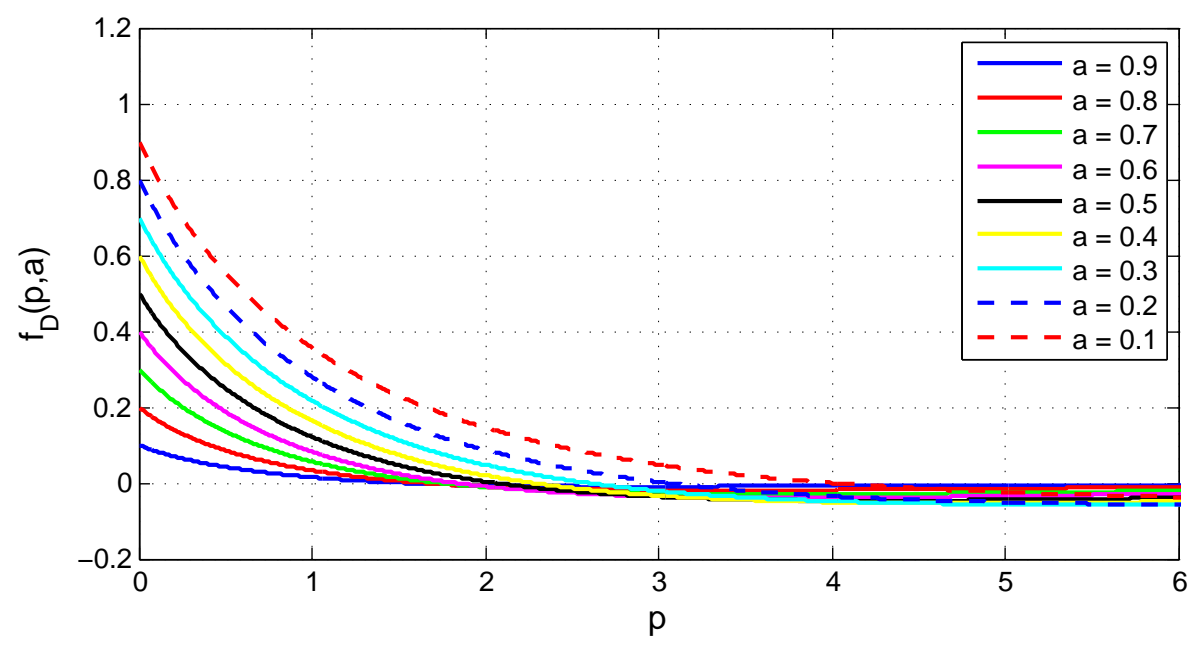

(a)

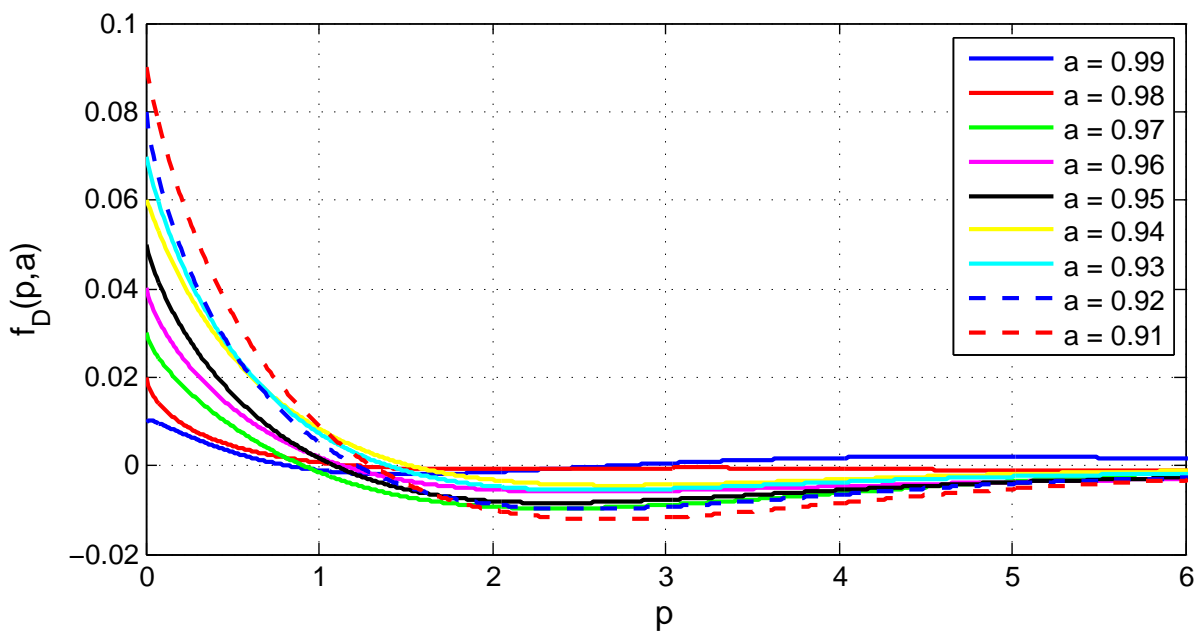

(b)

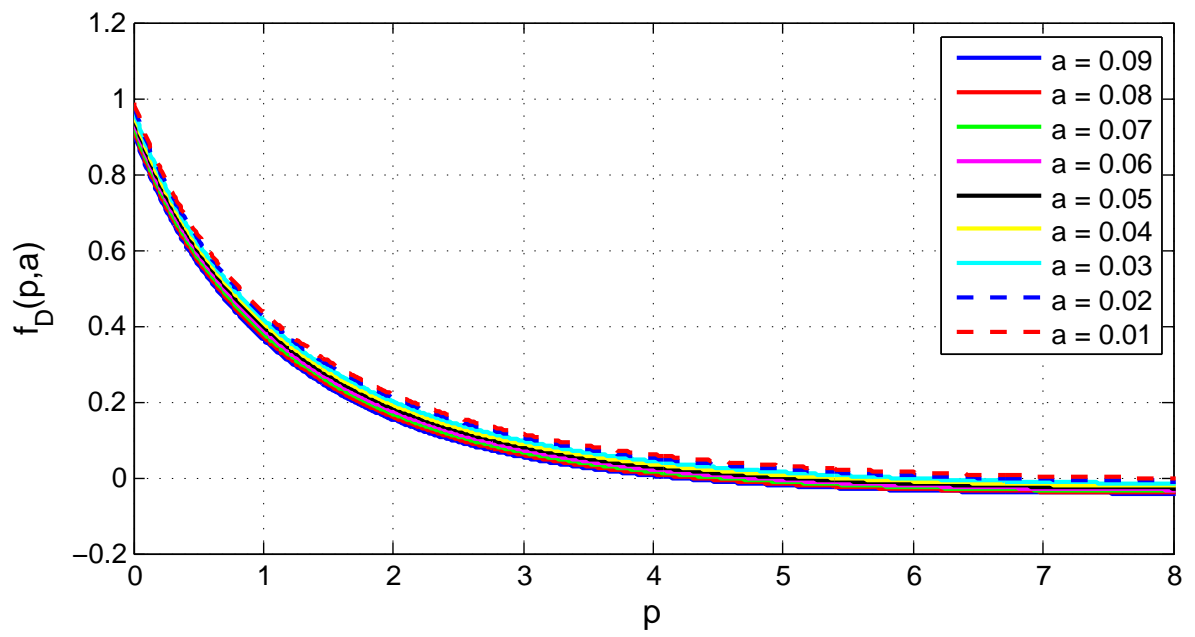

(c)

Fig. 8. MMSE difference function $f_{D}(p, a)$ for different values of $a=e_{i}^{2} / b_{i}^{2}$ for QPSK 


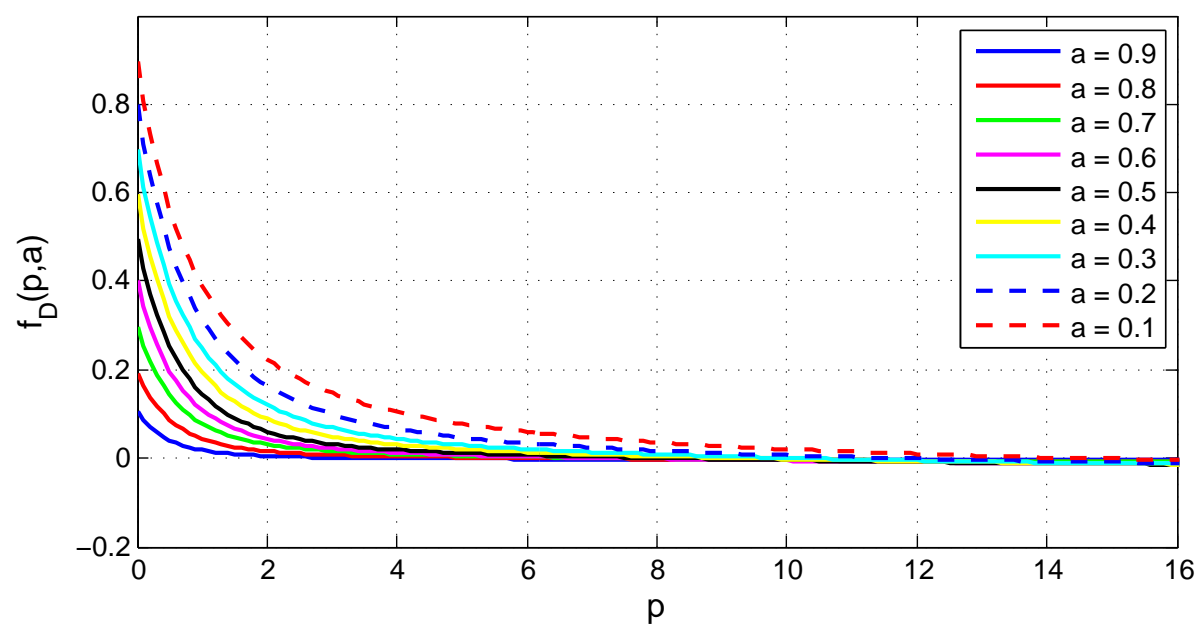

(a)

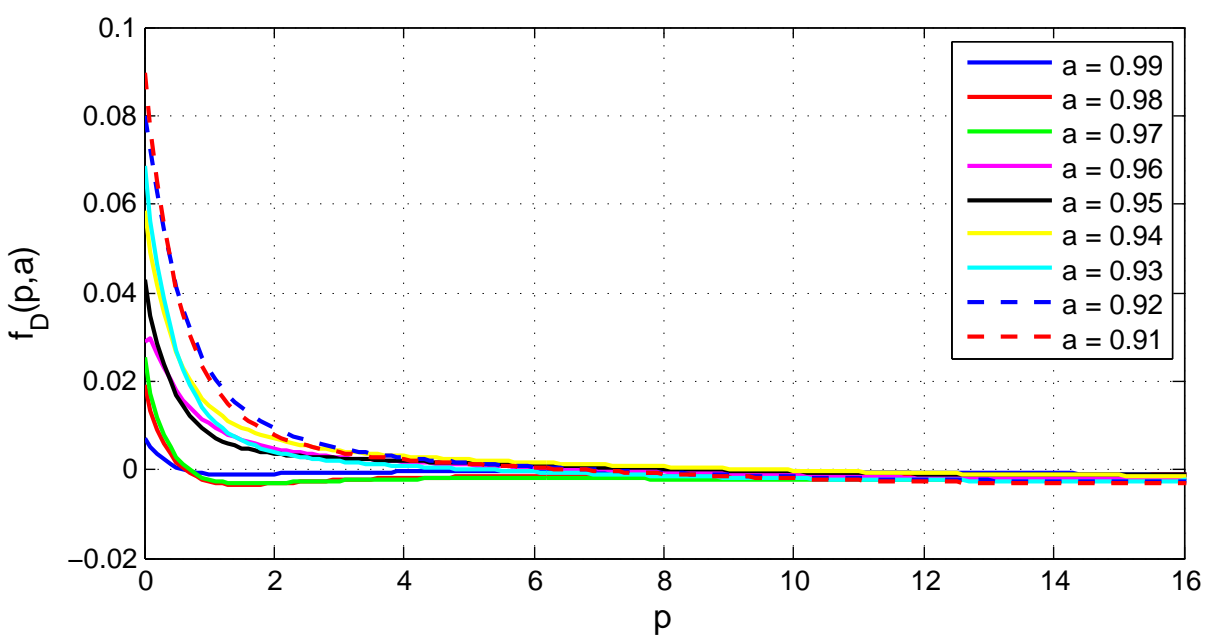

(b)

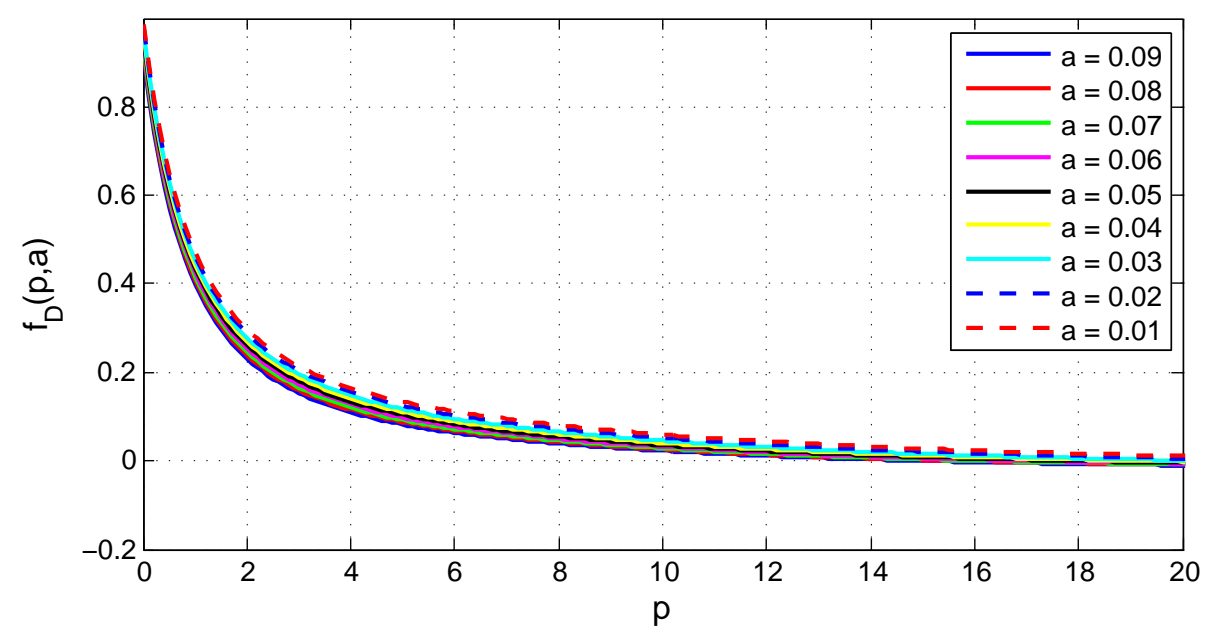

(c)

Fig. 9. MMSE difference function $f_{D}(p, a)$ for different values of $a=e_{i}^{2} / b_{i}^{2}$ for 16-QAM 


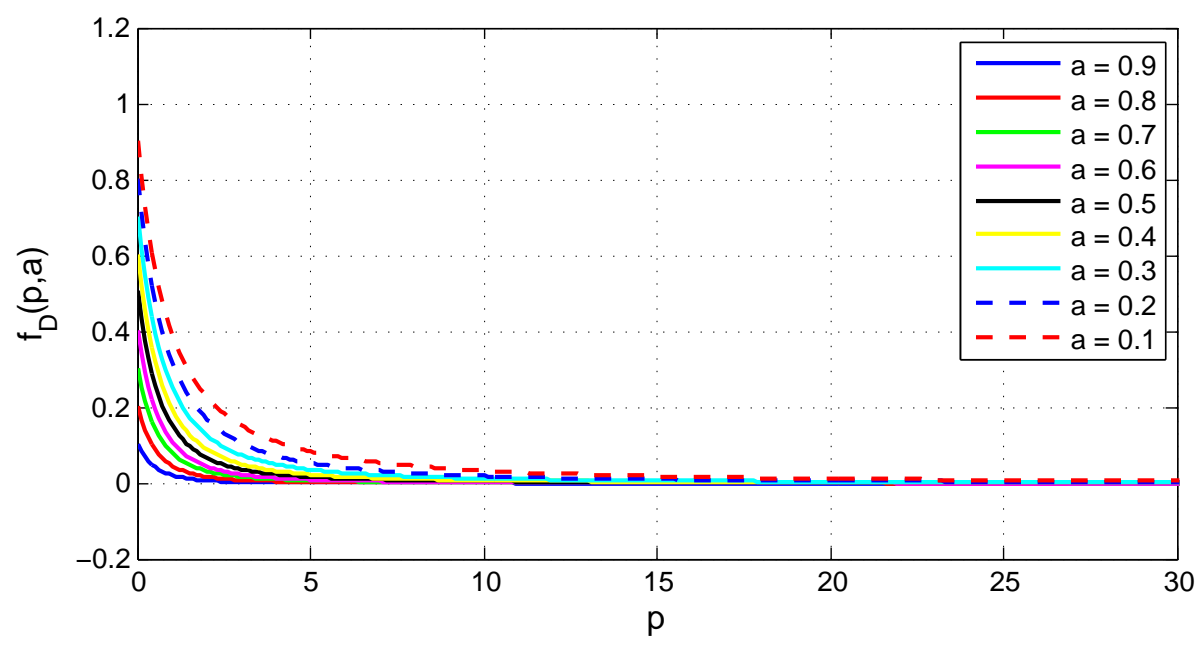

(a)

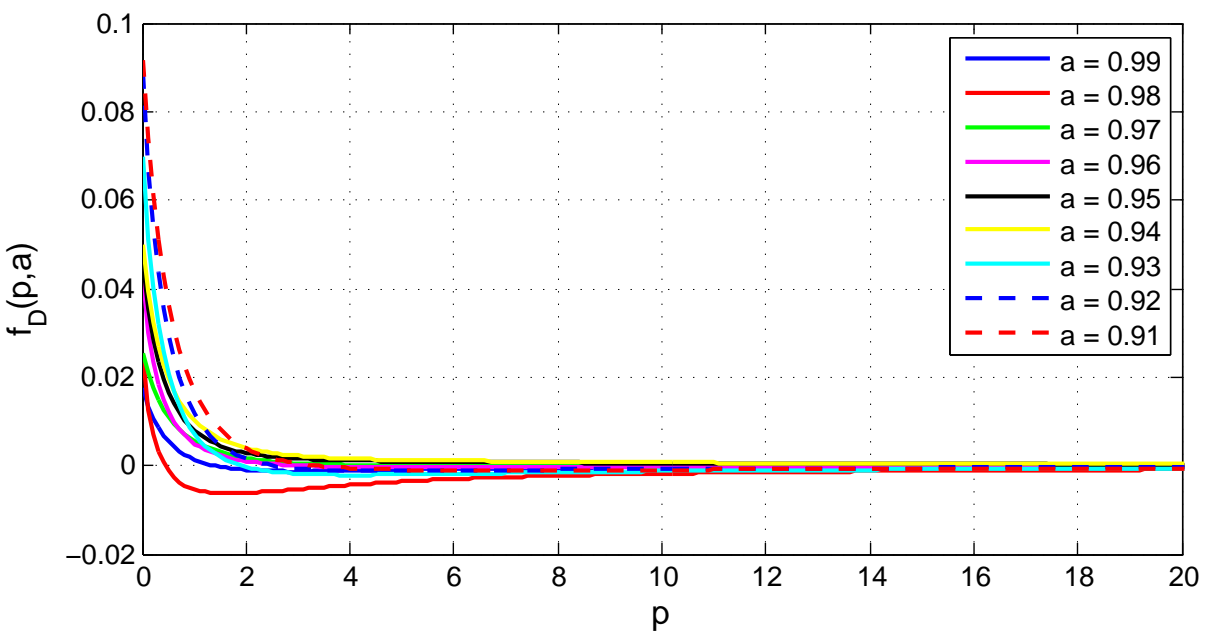

(b)

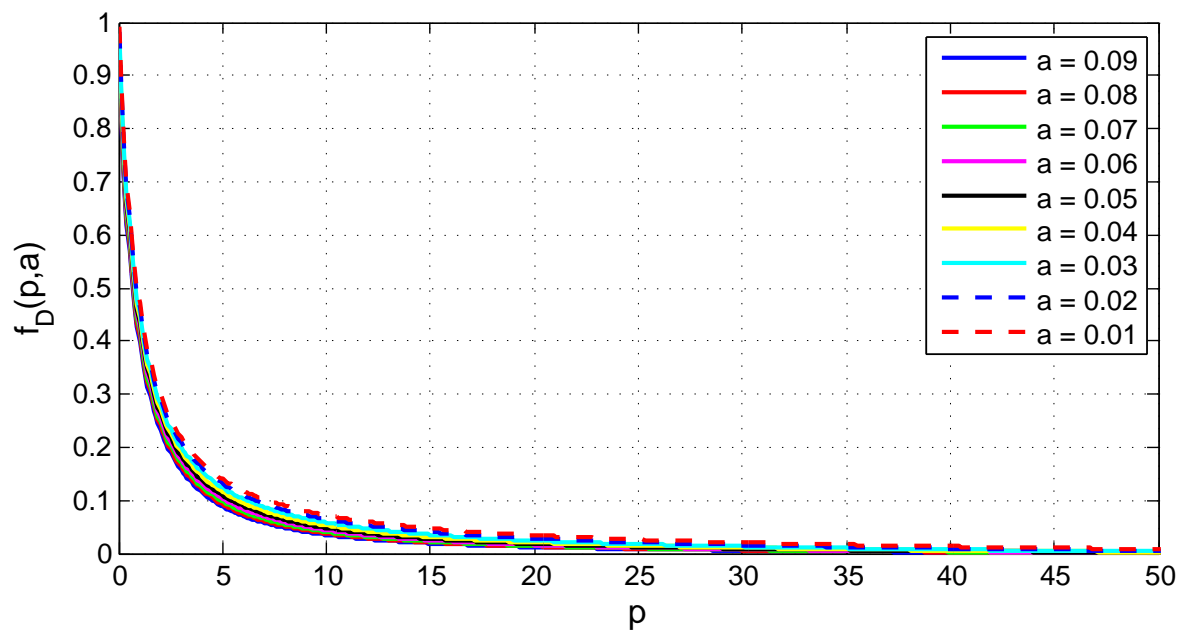

(c)

Fig. 10. MMSE difference function $f_{D}(p, a)$ for different values of $a=e_{i}^{2} / b_{i}^{2}$ for 64-QAM 